\title{
Non-Archimedean volumes of metrized nef line bundles
}

\author{
Sébastien Boucksom, Walter Gubler, and Florent Martin
}

\begin{abstract}
Let $L$ be a line bundle on a proper, geometrically reduced scheme $X$ over a non-trivially valued nonArchimedean field $K$. Roughly speaking, the non-Archimedean volume of a continuous metric on the Berkovich analytification of $L$ measures the asymptotic growth of the space of small sections of tensor powers of $L$. For a continuous semipositive metric on $L$ in the sense of Zhang, we show first that the non-Archimedean volume agrees with the energy. The existence of such a semipositive metric yields that $L$ is nef. A second result is that the non-Archimedean volume is differentiable at any semipositive continuous metric. These results are known when $L$ is ample, and the purpose of this paper is to generalize them to the nef case. The method is based on a detailed study of the content and the volume of a finitely presented torsion module over the (possibly non-Noetherian) valuation ring of $K$.
\end{abstract}

Keywords. Non-Archimedean geometry; Berkovich space; volume; nef line bundle

2020 Mathematics Subject Classification. 32P05; 14G22; 32U15; 32W20

[Français]

Volumes non-archimédiens des fibrés en droites nef métrisés

Résumé. Soit $L$ un fibré en droites sur un schéma propre et géométriquement réduit $X$ défini sur un corps $K$ muni d'une valuation non-triviale non-archimédienne. Grosso modo, le volume non-archimédien d'une métrique continue sur l'analytifié de Berkovich de $L$ mesure la croissance asymptotique de l'espace des petites sections des puissances tensorielles de $L$. Pour une métrique continue semi-positive sur $L$ au sens de Zhang, nous montrons tout d'abord que le volume non-archimédien coïncide avec l'énérgie. L'existence d'une telle métrique semi-positive impose le caractère nef de $L$. La différentiabilité du volume non-archimédien en toute métrique continue semi-positive constitue un deuxième résultat. Ces résultats sont connus lorsque $L$ est ample et l'objectif de cet article est de les généraliser au cas nef. La méthode s'appuie sur une étude détaillée du contenu et du volume d'un module de torsion de présentation finie sur l'anneau (éventuellement non noethérien) de valuation de $K$.

Received by the Editors on November 16, 2020, and in final form on May 25, 2021.

Accepted on August 18, 2021.

Sébastien Boucksom

Centre de Mathématiques Laurent Schwartz, Ecole Polytechnique and CNRS, Institut Polytechnique de Paris

e-mail: sebastien.boucksom@polytechnique.edu

Walter Gubler

W. Gubler, Mathematik, Universität Regensburg, 93040 Regensburg, Germany

e-mail: walter.gubler@mathematik.uni-regensburg.de

Florent Martin

F. Martin, Mathematik, Universität Regensburg, 93040 Regensburg, Germany

e-mail: florent.guy.martin@gmail.com

Sébastien Boucksom was partly supported by the ANR project GRACK. Walter Gubler and Florent Martin were supported by the collaborative research center SFB 1085 funded by the Deutsche Forschungsgemeinschaft.

(C) by the author(s)

This work is licensed under http://creativecommons.org/licenses/by-sa/4.0/ 


\section{Contents}

1. Introduction. . . . . . . . . . . . . . . . . . . . . . . . . . . . . . . . 2

2. Preliminaries . . . . . . . . . . . . . . . . . . . . . . . . . . . . 5

3. Volumes on torsion schemes . . . . . . . . . . . . . . . . . . . . . . . . . 14

4. Volume formulas for nef models and semipositive metrics $\quad$. . . . . . . . . . . . . . . . . . 21

5. Differentiability of non-Archimedean volumes . . . . . . . . . . . . . . . . . . . . . 26

6. Orthogonality property

Appendix A. Asymptotical functions .

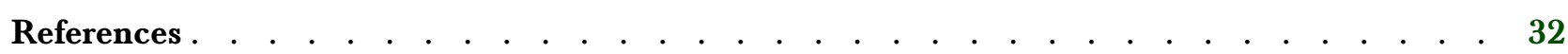

\section{Introduction}

The volume is an important invariant in algebraic geometry measuring asymptotically the size of the space of global sections. For a line bundle $L$ on an $n$-dimensional projective variety $Y$ over an algebraically closed field $k$, it is given by

$$
\operatorname{vol}(L):=\limsup _{m \rightarrow \infty} \frac{h^{0}(Y, m L)}{m^{n} / n !} .
$$

Here and in the following, we use additive notation for line bundles and metrics. The volume has many nice properties like continuity and differentiability for which we refer to Lazersfeld's books [Laz04a] and [Laz04b]. In Arakelov geometry, there is a similar invariant called the arithmetic volume. It measures asymptotically the size of the number of small sections. For a projective arithmetic variety $\mathscr{X}$ of relative dimension $n$ over the ring of integers $\mathcal{O}_{F}$ of a number field $F$ and a line bundle $\mathscr{L}$ on $\mathscr{X}$ endowed with Hermitian metrics $\phi_{v}$ for each Archimedean place $v$, it is defined by

$$
\widehat{\operatorname{vol}}(\bar{L}):=\limsup _{m \rightarrow \infty} \frac{\log \#\left\{s \in H^{0}(\mathscr{X}, m \mathscr{L})\left|\|s\|_{m \phi_{v}} \leq 1, \forall v\right| \infty\right\}}{m^{n+1} /(n+1) !}
$$

where $\|s\|_{m \phi_{v}}$ is the supremum norm of $s$ associated to the metric $m \phi_{v}$. The arithmetic volume behaves similarly to the classical volume above, see Chen [Che08], Moriwaki [Mor09] and Yuan [Yua08]. In the philosophy of Lang, Néron and Weil, arithmetic invariants are always influenced by local invariants depending only on a single place of the number field $F$. The main object of study of this paper is a local variant of the arithmetic volume which we study over any non-Archimedean field. For its relation to the global arithmetic volume in case of a number field, we refer to $\left[\mathrm{BGJ}^{+} 20\right.$, Remark 4.1.7].

In the following, $K$ is a non-Archimedean field, i.e. a field $K$ endowed with a complete non-Archimedean absolute value $|\cdot|$, assumed to be non-trivially valued, here and throughout the paper. The valuation ring of $K$ is denoted by $K^{\circ}$, and the residue field by $\tilde{K}$. We consider a line bundle $L$ on an $n$-dimensional reduced proper scheme $X$ over $K$. Then the Berkovich analytification $X^{\text {an }}$ is compact and we consider a metric $\phi$ on $L$ which is continuous with respect to the Berkovich topology. We denote the associated supremum norm on $H^{0}(X, L)$ by $\|\cdot\|_{\phi}$. Using [BE21, §2.1], we note that $\|\cdot\|_{\phi}$ induces a canonical norm $\operatorname{det}\left(\|\cdot\|_{\phi}\right)$ on the one-dimensional $K$-vector space $\operatorname{det}\left(H^{0}(X, L)\right)$. Since a norm on a one-dimensional vector space 
is unique up to scaling, for any other continuous metric $\psi$ of $L$, we get a well-defined positive number $\operatorname{det}\left(\|\cdot\|_{\phi}\right) / \operatorname{det}\left(\|\cdot\|_{\psi}\right)$. Following [BE21, §2.3, §9.2], we define our local volume by

$$
\operatorname{vol}(L, \phi, \psi):=\limsup _{m \rightarrow \infty} \frac{n !}{m^{n+1}} \cdot \log \left(\operatorname{det}\left(\|\cdot\|_{m \psi}\right) / \operatorname{det}\left(\|\cdot\|_{m \phi}\right)\right)
$$

We call it here the non-Archimedean volume of $L$ with respect to $(\phi, \psi)$. In contrast to the global case, it is a relative notion which is only well-defined with respect to a pair of metrics $(\phi, \psi)$. For more details, we refer to $\S 2.6$. Non-Archimedean volumes were first introduced by Kontsevich and Tschinkel in [KT02] and they proposed their differentiability. The main result of this paper will show that this is true over any non-Archimedean field. Chen and Maclean [CM15] studied a variant of a local volume and their results yield that the limsup in (1.1) is in fact a limit if $X$ is geometrically reduced (see [BE21, Theorem 9.8]).

To describe our first result, we recall from algebraic geometry that the volume of a nef line bundle

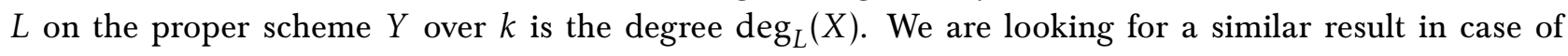
non-Archimedean volumes of the line bundle $L$ on an $n$-dimensional geometrically reduced proper scheme $X$ over any non-Archimedean field $K$. Let $\phi_{1}, \phi_{2}$ be continuous metrics of the line bundle $L$ which are semipositive in the sense of Zhang [Zha95]. We recall semipositivity in $\S 2.3$ and $\S 2.4$. The analogue of the degree in the relative setting is the energy

$$
\mathrm{E}\left(L, \phi_{1}, \phi_{2}\right):=\frac{1}{n+1} \sum_{j=0}^{n} \int_{X^{\text {an }}}\left(\phi_{1}-\phi_{2}\right)\left(d d^{c} \phi_{1}\right)^{j} \wedge\left(d d^{c} \phi_{2}\right)^{n-j} .
$$

On the right-hand side, we use the Monge-Ampère measures $\left(d d^{c} \phi_{1}\right)^{j} \wedge\left(d d^{c} \phi_{2}\right)^{n-j}$ on the Berkovich space $X^{\text {an }}$ introduced by Chambert-Loir [CL06]. We refer to $\S 2.4$ for details about these Radon measures including a proof of locality principle. The energy was introduced in non-Archimedean geometry in [BFJ15], we recall the basic properties in $\S 2.5$.

Theorem 1.1. Let $L$ be a line bundle on a reduced proper scheme $X$ over a non-Archimedean field $K$. If $\phi_{1}$, $\phi_{2}$ are two continuous semipositive metrics on $L^{\text {an }}$, then

$$
\operatorname{vol}\left(L, \phi_{1}, \phi_{2}\right)=\mathrm{E}\left(L, \phi_{1}, \phi_{2}\right) \text {. }
$$

In the corresponding Archimedean situation and for $L$ (nef and) big, this was shown in [BB10, Theorem A]. For $K$ discretely valued, the theorem was proved in $\left[\mathrm{BGJ}^{+} 20\right.$, Theorem A]. For $L$ ample and $K$ any nonArchimedean field, this is a result given in [BE21, Theorem A]. We generalize it here in Theorem 4.6 to any (nef) line bundle in case of an arbitrary non-Archimedean field $K$.

The main result of our paper is the following differentiability of the non-Archimedean volume.

Theorem 1.2. Let $L$ be a line bundle on the $n$-dimensional proper, geometrically reduced scheme $X$ over a nonArchimedean field K. Let $\phi$ be a continuous semipositive metric on $L$ and let $f: X^{\text {an }} \rightarrow \mathbb{R}$ be continuous. Then $\operatorname{vol}(L, \phi+t f, \phi)$ is differentiable at $t=0$ and

$$
\left.\frac{d}{d t}\right|_{t=0} \operatorname{vol}(L, \phi+t f, \phi)=\int_{X^{\text {an }}} f\left(d d^{c} \phi\right)^{n} .
$$

This formula is the non-Archimedean analogue of [BB10, Theorem B], and was proposed by Kontsevich and Tschinkel [KT02, §7.2]. Differentiability of arithmetic volumes was proven by Yuan (see [Yua08] and [Che11, $\S 4.4])$. In the case of a discretely valued field $K$, Theorem 1.2 was shown in [BGJ ${ }^{+} 20$, Theorem B]. For an ample line bundle, this was generalized in [BGM20, Theorem A] for any non-Archimedean base field $K$. We will deduce from it the more general Theorem 1.2 using the additional tools described below. This will be done in Theorem 5.3.

The proofs of Theorem 1.1 and Theorem 1.2 are similar, but for the latter additional problems arise from leaving the nef cone. Our arguments were inspired by the techniques for the proofs of the arithmetic HilbertSamuel theorem by Abbes and Bouche [AB95] and of a general equidistribution result by Yuan [Yua08]. Our 
proofs follow the overall plan in $\left[\mathrm{BGJ}^{+} 20\right]$ for the same statements in the DVR case, but we have to adapt it here at several places to deal with the non-Noetherian situation.

A crucial tool in the proofs of Theorem 1.1 and Theorem 1.2 is the volume of a line bundle $L$ over an $n$-dimensional finitely presented projective torsion scheme $Y$ over $K^{\circ}$ which we will introduce in Section 3 . It follows from the direct image theorem (see Ullrich [Ull95, Theorem 3.5]) that $H^{q}(Y, L)$ is a finitely presented torsion $K^{\circ}$-module $M$ and we define $h^{q}(Y, L):=\mathrm{c}(M)$. Here, $\mathrm{c}(M)$ is the content of a finitely presented torsion $K^{\circ}$-module $M$ which is a generalization of the length to our non-Noetherian situation and which was already considered by Scholze [Sch13], Temkin [Tem16] and in [BE21] (see § 2.2 for details). We will see in $\S 3.1$ that the invariants $h^{q}(Y, L)$ share many properties of the usual Hilbert-Samuel theory. Influenced by a similar construction in algebraic geometry by Küronya [Kür06], we define the q-th asymptotic cohomological functions by

$$
\hat{h}^{q}(Y, L):=\limsup _{m \rightarrow \infty} \frac{h^{q}(Y, m L)}{m^{n} / n !} .
$$

For $q=0$, we call it the volume of $L$ and we $\operatorname{set} \operatorname{vol}(Y, L):=\hat{h}^{0}(Y, L)$. In Section 3, we show the asymptotic cohomological functions are continuous and homogeneous of degree $n$.

Another basic ingredient is the following Hilbert-Samuel type formula: let $\mathscr{X}$ be a projective flat scheme over $K^{\circ}$ with generic fiber $X:=\mathscr{X} \otimes_{K^{\circ}} K$ and let $n:=\operatorname{dim}(X)$. Let $\mathscr{L}$ be a nef line bundle on $\mathscr{X}$ with associated model metric $\phi_{\mathscr{L}}$ on $L:=\left.\mathscr{L}\right|_{X}$. Let $D$ be a vertical effective Cartier divisor on $\mathscr{X}$ with associated model function $\phi_{D}:=\phi_{\mathcal{O}(D)}$. Then we have

$$
\operatorname{vol}(D, \mathscr{L})=\int_{X^{\text {an }}} \phi_{D}\left(d d^{c} \phi \mathscr{L}\right)^{n}
$$

Based on crucial results for the Deligne pairing in [BE21], this formula was proven in [BGM20, Theorem 2.4] for $\mathscr{L}$ ample. In Proposition 4.2, the continuity of the volume on the left-hand side allows us to generalize (1.3) for nef line bundles $\mathscr{L}$. Then both Theorem 1.1 and Theorem 1.2 follow from (1.3) by using a variant of Yuan's filtration argument.

In Section 6, we give two applications of the above theorems. We suppose that $L$ is a line bundle on a proper geometrically reduced scheme $X$ over $K$. We assume $\psi$ is a continuous metric on $L$ such that the semipositive envelope

$$
\mathrm{P}(\psi):=\sup \left\{\phi \mid \phi \text { is a continuous semipositive metric on } L^{\text {an }} \text { and } \phi \leq \psi\right\}
$$

is a continuous metric on $L$. This is expected to hold for all normal projective varieties and semiample line bundles (see [BE21, Conjecture 7.31]). Then we show in Corollary 6.5 that

$$
\operatorname{vol}(L, \phi, \psi)=\mathrm{E}(L, \mathrm{P}(\phi), \mathrm{P}(\psi))
$$

where $\psi$ is another continuous metric on $L$ with $\mathrm{P}(\psi)$ continuous. The formula (1.4) was shown in [BE21, Corollary 9.16] for $L$ ample. For $\phi$ as above, we will prove in Theorem 6.7 the orthogonality property

$$
\int_{X^{\text {an }}}(\mathrm{P}(\phi)-\phi)\left(d d^{c} \mathrm{P}(\phi)\right)^{n}=0 .
$$

This is a crucial property in the proof of the existence of solutions of non-Archimedean Monge-Ampère equations, see [BFJ15].

We repeat here that we assume in the whole paper that the absolute value of the non-Archimedean field $K$ is non-trivial. The reason is that we want to work with semipositive metrics in the sense of Zhang. Note that in the trivially valued case, every line bundle on a proper variety has only one model metric and hence there is also a unique semipositive metric. To get a rich theory in the trivially valued case, one has to use a different semipositivity notion as for example in [BE21] or [BJ18]. Still, our results can also be applied in the trivially valued case using that the Monge-Ampère measures, the energy and the non-Archimedean volume are compatible with base change (see [BE21]). 


\section{Acknowledgments}

We are grateful to José Burgos Gil, Antoine Ducros, Dennis Eriksson, Philipp Jell, Mattias Jonsson, Klaus Künnemann and Joe Rabinoff for many helpful discussions in relation to this work. We thank the referee for the careful reading and helpful comments.

\section{Notations and conventions}

The set of natural numbers $\mathbb{N}$ includes 0 . The rings in this paper are usually commutative and with 1 . If $A$ is such a ring and $a \in A$, then $(a)$ denotes the ideal of $A$ generated by $a$. If $P$ is an abelian group, then $P_{A}:=P \otimes_{\mathbb{Z}} A$ denotes the $A$-module obtained by base change.

As a base field, we consider usually a non-Archimedean field $K$. This means in the whole paper that $K$ is endowed with a non-Archimedean complete absolute value $|\cdot|$ which is non-trivial. We use $K^{\circ}:=\{\alpha \in K|1 \geq| \alpha \mid\}$ for the valuation ring, $K^{\circ \circ}:=\{\alpha \in K|1>| \alpha \mid\}$ for the maximal ideal in $K^{\circ}$ and $\tilde{K}:=K^{\circ} / K^{\circ \circ}$ for the residue field. If $M$ is a finitely presented torsion module over $K^{\circ}$, then we denote by $c(M)$ the content of $M$. This is a generalization of the length in our non-Noetherian situation which we will introduce in $\S 2.2$.

Let $X$ be a scheme. If $\mathcal{F}$ is a coherent sheaf on a scheme $X$ and $D$ is a Cartier divisor on $X$, we write $\mathcal{F}(D)$ for $\mathcal{F} \otimes_{\mathcal{O}_{X}} \mathcal{O}_{X}(D)$. We will use additive notation for line bundles. If $L, M$ are line bundles on $X$, then $L+M$ denotes the tensor product of the line bundles $L$ and $M$. For $m \in \mathbb{Z}$, we denote the $m$-th tensor power of $L$ by $m L$.

Let $X$ be an $n$-dimensional proper scheme over the field $F$. For line bundles $L_{1}, \ldots, L_{n}$, we denote the degree of $X$ with respect to $L_{1}, \ldots, L_{n}$ by $\operatorname{deg}_{L_{1}, \ldots, L_{n}}(X)$. It is given by the intersection number

$$
\operatorname{deg}_{L_{1}, \ldots, L_{n}}(X)=\mathrm{c}_{1}\left(L_{1}\right) \cdots \mathrm{c}_{1}\left(L_{n}\right) \cdot[X] .
$$

If all line bundles agree with $L$, then we use $\operatorname{deg}_{L}(X):=\operatorname{deg}_{L_{1}, \ldots, L_{n}}(X)$ as a shorthand. We set

$$
h^{q}(X, L):=\operatorname{dim} H^{q}(X, L) .
$$

If $X$ is a proper scheme over the non-Archimedean field $K$, then we denote by $X^{\text {an }}$ its Berkovich analytification. A continuous metric $\phi$ on $L$ means that $\phi$ is continuous with respect to the Berkovich topology. Again, we use additive notation for metrics which means that the tensor metric of metrics $\phi$ and $\psi$ is denoted by $\phi+\psi$ (see 2.10 for more). The associated norm on fibers of $L$ is denoted by $|\cdot|_{\phi}$ and $\|\cdot\|_{\phi}$ denotes the supremum seminorm on the space $H^{0}(X, L)$ of global sections. Usually, we consider $\|\cdot\|_{\phi}$ if $X$ is reduced, and it is then a norm.

\section{Preliminaries}

In this section, we fix an arbitrary non-Archimedean field $K$. We collect here some background results on the content of a module, models, Monge-Ampère measures, energy and non-Archimedean volumes.

\section{1. $\mathbb{Q}$-line bundles and positivity in the real Picard group}

In this subsection, we consider a proper, finitely presented scheme $Y$ over $K^{\circ}$. We will recall and fix notations about line bundles. The special fiber of $Y$ is $Y_{s}:=Y \otimes_{K^{\circ}} \tilde{K}$.

A $\mathbb{Q}$-line bundle on $Y$ is a pair $(M, m)$ with $M$ a line bundle on $Y$ and $m \in \mathbb{N}_{>0}$. The set of morphisms between $\mathbb{Q}$-line bundles $(M, m)$ and $(N, n)$ is given as the inductive limit

$$
\underset{k \in \mathbb{N}_{>0}}{\lim } \operatorname{Hom}(k n M, k m N) \text {. }
$$


The tensor product of line bundles induces a tensor product of $\mathbb{Q}$-line bundles. As usual, we denote the $\mathbb{Q}$-line bundle $(M, m)$ by $L$ and we will say that $m L$ is (induced by) an honest line bundle $M$. Note that $L=(M, m)$ induces a canonical element

$$
L \otimes 1:=M \otimes \frac{1}{m} \in \operatorname{Pic}(Y)_{\mathbb{Q}}:=\operatorname{Pic}(Y) \otimes_{\mathbb{Z}} \mathbb{Q} .
$$

We call $L$ nef (resp. ample) if $M$ is a nef (resp. ample) line bundle on $Y$. These notions are well-defined for elements of $\operatorname{Pic}(Y)_{\mathbb{Q}}:=\operatorname{Pic}(Y) \otimes_{\mathbb{Z}} \mathbb{Q}$.

Definition 2.1. By multilinearity, we extend the intersection pairing to define an intersection number $M \cdot C \in \mathbb{R}$ for any $M \in \operatorname{Pic}(Y)_{\mathbb{R}}$ and any closed curve $C$ in $Y_{s}$. Then $M \in \operatorname{Pic}(Y)_{\mathbb{R}}:=\operatorname{Pic}(Y) \otimes_{\mathbb{Z}} \mathbb{R}$ is called nef if $M \cdot C \geq 0$ for any closed curve $C$ in $Y_{s}$.

We call $M \in \operatorname{Pic}(Y)_{\mathbb{R}}$ ample if there are ample $L_{1}, \ldots, L_{r} \in \operatorname{Pic}(Y)$ and $\lambda_{1}, \ldots, \lambda_{r}>0$ with $M=\sum_{i=1}^{r} \lambda_{i} L_{i}$ for some non-zero $r \in \mathbb{N}$.

The definition of ample is consistent for $\mathbb{Q}$-line bundles, by the Nakai-Moishezon criterion applied to $Y_{S}$. Obviously, ample implies nef.

Proposition 2.2. Let $A \in \operatorname{Pic}(Y)_{\mathbb{R}}$ be ample and $M \in \operatorname{Pic}(Y)_{\mathbb{R}}$ be nef. Then $A+M$ is ample.

Proof. The important observation is that $L \in \operatorname{Pic}(Y)$ is nef (resp. ample) if and only if the restriction $\left.L\right|_{Y_{s}}$ to the special fiber is nef (resp. ample). This is by definition in the nef case, and it follows from [Gro66, Corollaire 9.6.5] in the ample case.

Assume first that $A \in \operatorname{Pic}(Y)$ is ample and $M \in \operatorname{Pic}(Y)$ is nef. Then it is well-known that $\left.A\right|_{Y_{s}}+\left.M\right|_{Y_{s}}$ is ample and hence $A+M$ is ample. This implies the claim for $\mathbb{Q}$-line bundles.

To show the first claim in general, it is enough to show that $A+M$ is ample for $A \in \operatorname{Pic}(Y)$ ample and $M \in \operatorname{Pic}(Y)_{\mathbb{R}}$ nef. The existence of the ample line bundle $A$ yields that $Y$ is projective. We conclude that any element in $\operatorname{Pic}(Y)$ is the difference of two ample classes [Gro61, Corollaire 4.5.8] and hence we may assume that $M=\sum_{i=1}^{r} \lambda_{i} H_{i}$ for $H_{i} \in \operatorname{Pic}(Y)$ ample and $\lambda_{i} \in \mathbb{R}$. For $\delta>0$ sufficiently small, we choose $\rho_{i} \in \mathbb{Q}$ with $\lambda_{i}<\rho_{i}<\lambda_{i}+\delta$. Since all $H_{i}$ are ample and $M$ is nef, we deduce that $N=\sum_{i} \rho_{i} H_{i}$ is nef. Since the ample cone on $Y_{S}$ is open, we can choose $\delta \in \mathbb{Q}_{>0}$ so small that the restriction of $A-\delta \sum_{i} H_{i}$ to $Y_{s}$ is ample. By the observation at the beginning, we get that $A-\delta \sum_{i} H_{i}$ is ample and hence $A-\delta \sum_{i} H_{i}+N$ is ample by the claim for $\mathbb{Q}$-line bundles which we already have shown. Note that

$$
A+M=A+\sum_{i=1}^{r} \lambda_{i} H_{i}=A-\delta \sum_{i=1}^{r} H_{i}+N+\sum_{i=1}^{r}\left(\lambda_{i}+\delta-\rho_{i}\right) H_{i}
$$

and hence $A+M$ is ample as $\lambda_{i}+\delta-\rho_{i}>0$.

Remark 2.3. Let $Y$ be a finitely presented projective scheme over $K^{\circ}$ and let $M$ be a nef element in $\operatorname{Pic}(Y)_{\mathbb{R}}$. Then there is a finite dimensional subspace $W$ of $\operatorname{Pic}(Y)_{\mathbb{Q}}$ such that $M$ is the limit of a sequence of ample elements in $W$. Indeed, the above proof shows that $M$ is the limit of nef elements $N_{k} \in\left\langle H_{1}, \ldots, H_{r}\right\rangle_{\mathbb{Q}}$ and hence $M$ is the limit of the ample classes $N_{k}+\frac{1}{k} A$.

Remark 2.4. For $M \in \operatorname{Pic}(Y)_{\mathbb{R}}$, it is clear that $M$ is nef if and only if the restriction of $M$ to the special fiber $Y_{S}$ is nef. The arguments in the proof of Proposition 2.2 show that $M$ is ample if and only if $\left.M\right|_{Y_{s}}$ is ample.

\subsection{Lattices and content}

We will introduce the content of a finitely presented $K^{\circ}$-module as a generalization of the length in our non-Noetherian situation. At the end, we will extend the content to the virtual quotient of two lattices in the same finite dimensional $K$-vector space. 
2.5. By [Sch13, Proposition 2.10 (i)], if $M$ is a finitely presented torsion module over $K^{\circ}$, then there is an integer $m$ and some $a_{1}, \ldots a_{m} \in K^{\circ \circ} \backslash\{0\}$ such that

$$
M \simeq K^{\circ} /\left(a_{1}\right) \oplus \cdots \oplus K^{\circ} /\left(a_{m}\right)
$$

and the quantities $m$ and $v\left(a_{i}\right)$ are independent of any choice up to reordering.

Definition 2.6. Let $M$ be a finitely presented torsion $K^{\circ}$-module and use the above decomposition. We define as in [BE21, §2.4] the content of $M$ to be

$$
\mathrm{c}(M)=\sum_{i=1}^{m} v\left(a_{i}\right) \in \mathbb{R}_{\geq 0} .
$$

In [Sch13], the content $\mathrm{c}(M)$ is called the length of $M$.

Remark 2.7. Recall from [BE21, Ex 2.19]) that $\mathrm{c}(M)$ agrees with the usual length in case of a discrete valuation with $v(\pi)=1$ for an uniformizing element $\pi$ of $K^{\circ}$.

Proposition 2.8. Let $0 \rightarrow M_{1} \rightarrow \cdots \rightarrow M_{m} \rightarrow 0$ be an exact sequence of finitely presented torsion $K^{\circ}{ }^{-}$modules. Then we have

$$
\sum_{i}(-1)^{i} \mathrm{c}\left(M_{i}\right)=0
$$

Proof. This is a consequence of [Sch13, Proposition 2.10].

2.9. A finitely generated $K^{\circ}$-submodule $\mathcal{V}$ of a finite dimensional $K$-vector space $V$ is called a lattice in $V$ if $\mathcal{V}$ generates $V$ as a $K$-vector space. If $\mathcal{V}_{1}, \mathcal{V}_{2}$ are lattices in $V$, then there is a lattice $\mathcal{V}$ of $V$ contained in $\mathcal{V}_{1} \cap \mathcal{V}_{2}$. Note that any finitely generated $K^{\circ}$-submodule $\mathcal{V}$ of $V$ is contained in a free $K^{\circ}$-submodule of finite rank and hence $\mathcal{V}$ is finitely presented over $K^{\circ}$ by using that $K^{\circ}$ is a coherent ring [Ul195, Proposition 1.6]. For $i=1,2$, it follows that $\mathcal{V}_{i} / \mathcal{V}$ is a finitely presented torsion module over $K^{\circ}$ and we define the content of the virtual $K^{\circ}$-module $\mathcal{V}_{1} / \mathcal{V}_{2}$ as

$$
\mathrm{c}\left(\mathcal{V}_{1} / \mathcal{V}_{2}\right):=\mathrm{c}\left(\mathcal{V}_{1} / \mathcal{V}\right)-\mathrm{c}\left(\mathcal{V}_{2} / \mathcal{V}\right)
$$

by using the content of finitely presented torsion modules over $K^{\circ}$ from Definition 2.6. Additivity of the content shows that this is a well-defined real number which might be negative.

\subsection{Models and metrics}

In this subsection, we consider a line bundle $L$ on a proper scheme $X$ over $K$. We will introduce models of $X$ and $L$ defined over the valuation ring $K^{\circ}$. We will see that a model of $L$ induces a metric on $L^{\text {an }}$. Such metrics are called model metrics. They were introduced by Zhang [Zha95] in Arakelov theory and they play a similar role in non-Archimedean geometry as smooth metrics in the Archimedean case.

2.10. As in [BE21, §5], we use the logarithmic notation for a metric on a line bundle $L$ on $X$ : a metric on $L$ is a function $\phi: L^{\text {an }} \rightarrow \mathbb{R}$ such that $|\cdot|_{\phi}:=e^{-\phi}$ induces a norm on the $\mathscr{H}(x)$-vector space $L \otimes_{X} \mathscr{H}(x)$ for every $x \in X^{\text {an }}$. Here, $\mathscr{H}(x)$ is the completed residue field of $x$ endowed with its canonical absolute value [Ber90, Remark 1.2.2]. The metric is called continuous if $\phi$ is continuous with respect to the Berkovich topology. If $\phi$ is a (continuous) metric on $\mathcal{O}_{X}$, then we identify $\phi$ with the (continuous) function $-\log |1|_{\phi}$ on $X^{\text {an }}$.

Definition 2.11. A model $\mathscr{X}$ of $X$ is a flat, proper scheme $\mathscr{X}$ over $K^{\circ}$ together with an identification of its generic fiber $\mathscr{X}_{\eta}:=\mathscr{X} \otimes_{K^{\circ}} K$ with $X$. There is a canonical reduction map red $\mathscr{X}: X^{\text {an }} \rightarrow \mathscr{X}_{s}$ to the special fiber $\mathscr{X}_{s}$ of $\mathscr{X}$ (see [GM19, Remark 2.3] and [GRW17, §2] for details). On closed points of $X$, the reduction is induced by the valuative criterion of properness, and hence coincides with the usual reduction modulo the maximal ideal of $K^{\circ}$.

We say that a model $\mathscr{X}$ is integrally closed in $X$ if for every affine open subset $\mathcal{U}$ of $\mathscr{X}$, the $\operatorname{ring} \mathcal{O}(\mathcal{U})$ is integrally closed in $\mathcal{O}\left(\mathcal{U}_{\eta}\right)$, see [Gro61, §6.3] for the integral closure of a scheme. 
A model $(\mathscr{X}, \mathscr{L})$ of $(X, L)$ is a model $\mathscr{X}$ of $X$ and a line bundle $\mathscr{L}$ on $\mathscr{X}$ together with an identification $\left.\mathscr{L}\right|_{\mathscr{X}_{\eta}} \simeq L$ compatible with the identification $\mathscr{X}_{\eta} \simeq X$. We call $\mathscr{L}$ a model of L determined on $\mathscr{X}$.

Remark 2.12. We say that the model $\mathscr{X}$ is dominated by a model $\mathscr{X}^{\prime}$ of $X$ if the identity on $X$ extends to a (unique) morphism $\mathscr{X}^{\prime} \rightarrow \mathscr{X}$ over $K^{\circ}$. This induces a partial order on the set of isomorphism classes of models of $X$. It is easy to show that the isomorphism classes of models of $X$ form a directed system with respect to this partial order.

If $X$ is projective, then it follows as in [Gub03, Proposition 10.5] that the projective models of $X$ are cofinal among all models of $X$.

Remark 2.13. We will frequently use in this paper that if $(\mathscr{X}, \mathscr{L})$ is a model of $(X, L)$, then $H^{0}(\mathscr{X}, \mathscr{L})$ is a lattice in $H^{0}(X, L)$. Indeed, it follows from the direct image theorem given in [Ull95, Theorem 3.5] that $H^{0}(\mathscr{X}, \mathscr{L})$ is a finitely presented $K^{\circ}$-module. Using that $K$ is a flat $K^{\circ}$-module, we deduce that $H^{0}(\mathscr{X}, \mathscr{L})$ is a lattice in $H^{0}(X, L)$.

2.14. Let $(\mathscr{X}, \mathscr{L})$ be a model of $(X, L)$. Then there is an associated metric $\phi_{\mathscr{L}}$ of $L$ determined as follows: for $x \in X^{\text {an }}$, pick an open subset $\mathcal{U}$ of $\mathscr{X}$ which contains $\operatorname{red}_{\mathscr{X}}(x)$ and which trivializes $\mathscr{L}$. Then $\mathscr{L}(\mathcal{U}) \cong \mathcal{O}(\mathcal{U})$ mapping $s$ to $\gamma$ and the metric is given in $x$ by $|s(x)|_{\phi_{\mathscr{S}}}=|\gamma(x)|$. This does not depend on the choice of the trivialization. It is clear that such metrics are continuous on $L^{\text {an }}$. We refer to [BE21, 5.3] and [GM19, §2] for more details.

2.15. A metric $\phi$ on $L$ is called a model metric if there is a non-zero $k \in \mathbb{N}$ and a model $\mathscr{L}$ of $k L$ such that $k \phi=\phi \mathscr{L}$. We can say that the model metric $\phi$ is given by the $\mathbb{Q}$-model $\mathscr{M}:=\frac{1}{k} \mathscr{L}$ of $L$ and we will denote it by $\phi_{\mathscr{M}}$. A model metric is called semipositive if $\mathscr{M}$ is nef. Then $L$ is nef by [GM19, 4.8]. Note that the model $\mathscr{L}$ and hence $\mathscr{M}$ is not unique, but nefness of $\mathscr{M}$ is independent of the choice. For this and more details about model metrics, we refer to [GM19].

If $L=\mathcal{O}_{X}$, then we identify a model metric $\phi$ with the model function $-\log |1|_{\phi}$. It follows from [Gub98, Theorem 7.12] that model functions form a dense $\mathbb{Q}$-subspace of the space of continuous real functions on $X^{\text {an }}$ and hence the set of model metrics of $L$ is dense in the space of continuous metrics on $L^{\text {an }}$ with respect to uniform convergence (see [GM19, Theorem 1.2] for a generalization).

Example 2.16. A Cartier divisor $D$ on a model $\mathscr{X}$ of $X$ is called vertical if $\left.D\right|_{X}$ is trivial. Then $\mathcal{O}(D)$ is a model of $\mathcal{O}_{X}$ and we define the model function associated to $D$ by

$$
\phi_{D}:=-\log |1|_{\mathcal{O}(D)} .
$$

Conversely, for any model $(\mathscr{X}, \mathscr{L})$ of $\left(X, \mathcal{O}_{X}\right)$, the trivial section 1 of $\mathcal{O}_{X}$ extends uniquely to a meromorphic section $s$ of $\mathscr{L}$ and then $D:=\operatorname{div}(s)$ is a vertical Cartier divisor on $\mathscr{X}$.

2.17. We say that a metric $\phi$ on $L$ is bounded if for any open subset $U$ which trivializes $L$ and any trivializing section $s \in H^{0}(U, L)$, the function $-\log |s|_{\phi}$ is locally bounded on $U^{\text {an }}$. Clearly, any continuous metric $\psi$ of $L$ is bounded and hence boundedness of $\phi$ is equivalent to $\phi-\psi$ bounded on $X^{\text {an }}$, by compactness of the latter. Bounded metrics are stable under tensor product, inverse and pull-back.

For a bounded metric $\phi$ on $L$, we define the sup-seminorm

$$
\|s\|_{\phi}:=\sup _{x \in X^{\text {an }}}|s(x)|_{\phi}
$$

of $s \in H^{0}(X, L)$. If $X$ is reduced, it is a norm on $H^{0}(X, L)$ (see [BE21, Lemma 4.1]).

For bounded metrics $\phi_{1}, \phi_{2}$ on $L$, we define the distance

$$
d\left(\phi_{1}, \phi_{2}\right):=\sup _{x \in X^{\text {an }}}\left|\left(\phi_{1}-\phi_{2}\right)(x)\right|<\infty .
$$

Recall that we view $\phi_{1}-\phi_{2}$ as the (bounded) function on $X^{\text {an }}$ given as $-\log |1|_{\phi_{1}-\phi_{2}}$. Clearly, $d$ is a metric on the space of bounded metrics of $L$ inducing the topology of uniform convergence. 
2.18. For a bounded metric $\phi$ on $L$, we will use the notation

$$
\widehat{H^{0}}(X, L, \phi):=\left\{s \in H^{0}(X, L) \mid\|s\|_{\phi} \leq 1\right\} \text {. }
$$

If the absolute value on $K$ is discrete and $X$ is reduced, then $\widehat{H^{0}}(X, L, \phi)$ is automatically a lattice in $H^{0}(X, L), c f$. [BE21, Lemma 1.29 (ii)]. In the case of a non-discrete valuation, this is not always true. However, it is true in case of a model metric $\phi_{\mathscr{L}}$ associated to a model $(\mathscr{X}, \mathscr{L})$ of $(X, L)$ with $\mathscr{X}$ integrally closed in $X$. Indeed, Lemma 2.19 below yields

$$
\widehat{H^{0}}(X, L, \phi \mathscr{L})=H^{0}(\mathscr{X}, \mathscr{L})
$$

and hence the claim follows from Remark 2.13.

Lemma 2.19. Assume that $\mathscr{X}$ is a model of $X$ which is integrally closed in $X$ and that $\mathscr{L}$ is a model of $L$ determined on $\mathscr{X}$, then

$$
H^{0}(\mathscr{X}, \mathscr{L})=\widehat{H^{0}}(X, L, \phi \mathscr{L})
$$

Proof. It is clear that $\subset$ holds in the claim. To prove the converse, we pick $s \in \widehat{H^{0}}(X, L, \phi \mathscr{L})$. Locally on an affine open subset $\mathcal{U}=\operatorname{Spec}(A)$ which trivializes $\mathscr{L}$, it is given by $\gamma \in A$. It follows from [CM18, Theorem 2.1] or [BE21, Theorem 4.15] that $A$ integrally closed in $A \otimes_{K^{\circ}} K$ yields that $A$ is the unit ball of $A \otimes_{K^{\circ}} K$ with respect to the sup-seminorm on $\operatorname{red}^{-1}\left(\mathcal{U}_{s}\right)$. Now by definition of a model metric and as $s \in \widehat{H^{0}}(X, L, \phi \mathscr{L})$, we know that $\gamma$ is in this unit ball and hence in $A$. This proves $s \in \widehat{H^{0}}(\mathscr{X}, \mathscr{L})$.

Lemma 2.20. Assume that the model $\mathscr{X}$ of $X$ is integrally closed in $X$. Let $D$ be a vertical Cartier divisor of $\mathscr{X}$. Then $D$ is effective if and only if $\phi_{D} \geq 0$.

Proof. We note that $D$ is effective if and only if the canonical meromorphic section $s_{D}$ of $\mathcal{O}(D)$ is a global section. Since $D$ is a vertical Cartier divisor, the restriction of $s_{D}$ to $X$ is a (nowhere vanishing) global section of $\mathcal{O}_{X}$ and hence the claim is a special case of Lemma 2.19 .

Lemma 2.21. Assume that $K$ is algebraically closed and that $X$ is reduced (resp. reduced and projective). Then models (resp. projective models) of $X$ which are integrally closed in $X$ are cofinal among models of $X$.

Proof. This follows from Remark 2.12 and the scheme-theoretic version of the reduced fiber theorem of Bosch-Lütkebohmert-Raynaud given in [BE21, Theorem 4.20].

\subsection{Continuous semipositive metrics and Monge-Ampère measures}

Let $X$ be a proper scheme over the non-Archimedean field $K$. Recall from 2.15 the definition of a semipositive model metric on a line bundle $L$ over $X$. To describe canonical metrics of arithmetic dynamical systems, Zhang [Zha95] introduced the following generalization.

Definition 2.22. A continuous metric $\phi$ on $L^{\text {an }}$ is called semipositive if $\phi$ is a uniform limit of semipositive model metrics of $L$ with respect to the distance of uniform convergence from 2.17.

If $L$ has a continuous semipositive metric, then $L$ is nef by 2.15. The converse is not known. Note that continuous semipositive metrics are closed under sum, pull-back with respect to morphisms of proper schemes over $K$, and uniform limits. This is easily seen from the fact that the first two properties also hold for model metrics. Recall that we use additive notation for metrics, so sum means the tensor metric.

The following Monge-Ampere measures were introduced by Chambert-Loir.

Proposition 2.23. There is a unique way to associate to a tuple of continuous semipositive metrics $\phi_{1}, \ldots, \phi_{n}$ on line bundles $L_{1}, \ldots, L_{n}$ on a proper scheme $X$ over a non-Archimedean field $K$ and to an effective $n$-dimensional cycle $Z$ on $X$ a positive Radon measure $\mu$ on $X^{\text {an }}$, formally denoted by $d d^{c} \phi_{1} \wedge \cdots \wedge d d^{c} \phi_{n} \wedge \delta_{Z}$, such that the following holds: 
(a) the measure $\mu$ is multilinear and symmetric in $\phi_{1}, \ldots, \phi_{n}$;

(b) the measure $\mu$ is additive in $Z$;

(c) if $f: X^{\prime} \rightarrow X$ is a morphism of proper schemes and if $Z^{\prime}$ is an $n$-dimensional cycle on $X^{\prime}$, then we have the projection formula

$$
f_{*}\left(d d^{c}\left(f^{*} \phi_{1}\right) \wedge \cdots \wedge d d^{c}\left(f^{*} \phi_{n}\right) \wedge \delta_{Z^{\prime}}\right)=d d^{c} \phi_{1} \wedge \cdots \wedge d d^{c} \phi_{n} \wedge \delta_{f_{*}} Z^{\prime} ;
$$

(d) $\mu$ depends continuously on the $\phi_{i}$ with respect to uniform convergence (and weak convergence of Radon measures);

(e) $\mu\left(X^{\mathrm{an}}\right)=\operatorname{deg}_{L_{1}, \ldots, L_{n}}(Z)$;

(f) the Radon measure $\mu$ is compatible with base change of non-Archimedean fields;

(g) assume that $K$ is algebraically closed, and that $\phi_{1}, \ldots, \phi_{n}$ are model metrics determined by line bundles $\mathscr{L}_{1}, \ldots, \mathscr{L}_{n}$ on a model $\mathscr{X}$ of $X$ with reduced special fiber $\mathscr{X}_{s}$. Then

$$
d d^{c} \phi_{1} \wedge \cdots \wedge d d^{c} \phi_{n}:=d d^{c} \phi_{1} \wedge \cdots \wedge d d^{c} \phi_{n} \wedge \delta_{X}=\sum_{Y} \operatorname{deg}_{\mathscr{L}_{1}, \ldots, \mathscr{L}_{n}}(Y) \delta_{\xi_{Y}},
$$

where $Y$ ranges over all irreducible components of $\mathscr{X}_{s}$ and where $\delta_{\xi_{Y}}$ is the Dirac measure at the unique point $\xi_{Y} \in X^{\text {an }}$ with reduction equal to the generic point of $Y$.

Properties (b), (c), (d), (f) and (g) characterize the positive Radon measures $\mu$ uniquely.

Chambert-Loir [CL06] obtained this in the case of a non-Archimedean field with a countable dense subset. In the case of any algebraically closed non-Archimedean field, this follows from [Gub10, Proposition 3.8]. The general case is easily deduced by base change. Alternatively, this follows from the local approach in [CD12], see [BE21, §8.1] for details.

Remark 2.24. Note that the wedge product and the $d d^{c}$ in the notation $d d^{c} \phi_{1} \wedge \cdots \wedge d d^{c} \phi_{n} \wedge \delta_{Z}$ are a priori purely formal. It is used to stress the analogy to the complex Monge-Ampère measures where $d d^{c} \phi=c_{1}(L, \phi)$ is the first Chern form. It was shown later in [CD12, §6] and in [GK17, Theorem 10.5] that the measures could really be understood as a product of $(1,1)$-currents similarly to the complex case.

Remark 2.25. A continuous metric $\phi$ on a line bundle $L$ over $X$ is called a DSP metric if there are continuous semipositive metrics $\phi_{1}, \phi_{2}$ on line bundles $L_{1}, L_{2}$ over $X$ such that $L=L_{1}-L_{2}$ and $\phi=\phi_{1}-\phi_{2}$. By multilinearity, we can uniquely extend the construction of $\mu=d d^{c} \phi_{1} \wedge \cdots \wedge d d^{c} \phi_{n} \wedge \delta_{Z}$ to DSP metrics $\phi_{1}, \ldots, \phi_{n}$. The resulting Radon measure is no longer positive, but still satisfies (a)-(g).

If $X$ is projective, every model metric on a line bundle over $X$ is a DSP metric. Indeed, we have seen in Remark 2.12 that every model metric is determined on a projective model $\mathscr{X}$, and every line bundle on $\mathscr{X}$ is a difference of two ample line bundles on $\mathscr{X}$ [Gro61, Corollaire 4.5.8].

We recall the following result of Yuan and Zhang.

Proposition 2.26. Let $\phi_{2}, \ldots, \phi_{n}$ be continuous semipositive metrics of line bundles on the projective scheme $X$ over K. Then

$$
(f, g) \mapsto \int_{X^{\text {an }}} f d d^{c} g \wedge d d^{c} \phi_{2} \wedge \cdots \wedge d d^{c} \phi_{n}
$$

defines a negative semidefinite symmetric bilinear form on the $\mathbb{Q}$-vector space of model functions on $X$.

Proof. By base change, we may assume that $K$ is algebraically closed. Note that the form is given as a limit of intersection numbers on models and then the claim from the local Hodge index theorem from [YZ17, Theorem 2.1]. We refer to [BFJ15, Propositions 2.20, 2.21] for the argument. 
It follows from Remark 2.24 that the construction of Monge-Ampère measures is local in the Berkovich topology. All results in [BFJ15, §5] still hold. For convenience of the reader, we state and prove the comparison principle. The complex analogue is due to Bedford and Taylor and, in the discretely valued case of residue characteristic 0 , it is given in [BFJ15, Corollary 5.3].

Proposition 2.27. Let $\phi, \psi$ be continuous semipositive metrics on $L^{\text {an }}$. Then we have

$$
\int_{\{\phi<\psi\}}\left(d d^{c} \phi\right)^{n} \geq \int_{\{\phi<\psi\}}\left(d d^{c} \psi\right)^{n}
$$

where $\{\phi<\psi\}:=\left\{x \in X^{\text {an }} \mid \phi(x)<\psi(x)\right\}$.

Proof. We will follow closely the arguments from the complex case given in [BB10, Corollary 2.4]. Let $\varepsilon>0$, then $\psi_{\varepsilon}:=\max (\phi, \psi-\epsilon)$ is a continuous semipositive metric by [GM19, Propositions 3.11, 3.12]. By Proposition 2.23(e), we have

$$
\int_{X^{\mathrm{an}}}\left(d d^{c} \phi\right)^{n}=\operatorname{deg}_{L}(X)=\int_{X^{\mathrm{an}}}\left(d d^{c} \psi_{\varepsilon}\right)^{n} .
$$

Note that $\psi_{\varepsilon}=\phi$ on the open subset $\{\phi>\psi-\varepsilon\}$ of $X^{\text {an }}$ and that $\psi_{\varepsilon}=\psi-\varepsilon$ on the open subset $\{\phi<\psi-\varepsilon\}$ of $X^{\text {an }}$. Since these open subsets are disjoint and since formation of the Monge-Ampère measure is local in the Berkovich topology (i.e. compatible with restriction to open subsets), we get

$$
\int_{X^{a n}}\left(d d^{c} \psi_{\varepsilon}\right)^{n} \geq \int_{\{\phi>\psi-\varepsilon\}}\left(d d^{c} \phi\right)^{n}+\int_{\{\phi<\psi-\varepsilon\}}\left(d d^{c} \psi\right)^{n} .
$$

The right-hand side is bounded below by

$$
\int_{X^{\text {an }}}\left(d d^{c} \phi\right)^{n}-\int_{\{\phi>\psi\}}\left(d d^{c} \phi\right)^{n}+\int_{\{\phi<\psi-\varepsilon\}}\left(d d^{c} \psi\right)^{n} .
$$

Combining (2.1), (2.2) and (2.3) and using monotone convergence for $\varepsilon \rightarrow 0$, we get the claim.

\subsection{Energy}

We recall here the definition of the energy relative to two semipositive continuous metrics on a line bundle. We will see that all relevant properties of the energy from [BFJ15, §6] hold over any non-Archimedean field $K$.

In this subsection, we consider a line bundle $L$ on a proper scheme $X$ over $K$ of dimension $n$.

Definition 2.28. The energy of two continuous semipositive metrics $\phi_{1}, \phi_{2}$ of $L$ is

$$
\mathrm{E}\left(L, \phi_{1}, \phi_{2}\right):=\frac{1}{n+1} \sum_{j=0}^{n} \int_{X^{\mathrm{an}}}\left(\phi_{1}-\phi_{2}\right)\left(d d^{c} \phi_{1}\right)^{j} \wedge\left(d d^{c} \phi_{2}\right)^{n-j} \in \mathbb{R} .
$$

Note that in $[\mathrm{BJ} 18, \S 3.8]$, the energy was normalized by dividing through $\operatorname{deg}_{L}(X)$ which makes perfect sense in the case of an ample line bundle. Here, we will be also interested in nef line bundles and so we omit this normalization.

Proposition 2.29. Let $\phi_{1}, \phi_{2}, \phi_{1}^{\prime}, \phi_{2}^{\prime}, \phi_{3}$ be continuous semipositive metrics of L. Let $d$ be the distance on the space of bounded metrics of $L$ introduced in 2.17. Then the following holds.

(a) $\mathrm{E}\left(L, \phi_{2}, \phi_{1}\right)=-\mathrm{E}\left(L, \phi_{1}, \phi_{2}\right)$ and $\mathrm{E}\left(L, \phi_{1}, \phi_{1}\right)=0$.

(b) If $\phi_{1} \leq \phi_{2}$, then $\mathrm{E}\left(L, \phi_{1}, \phi_{3}\right) \leq \mathrm{E}\left(L, \phi_{2}, \phi_{3}\right)$.

(c) The cocycle rule $\mathrm{E}\left(L, \phi_{1}, \phi_{2}\right)+\mathrm{E}\left(L, \phi_{2}, \phi_{3}\right)+\mathrm{E}\left(L, \phi_{3}, \phi_{1}\right)=0$ holds.

(d) For $a \in \mathbb{N}$, we have the homogenity $\mathrm{E}\left(a L, a \phi_{1}, a \phi_{2}\right)=a^{n+1} \mathrm{E}\left(L, \phi_{1}, \phi_{2}\right)$.

(e) $\mathrm{E}\left(L, \phi_{1}+c, \phi_{2}\right)=\mathrm{E}\left(L, \phi_{1}, \phi_{2}\right)+c \operatorname{deg}_{L}(X)$ for $c \in \mathbb{R}$. 
(f) $\mathrm{E}\left(L, \phi_{1}, \phi_{2}\right)$ is concave in $\phi_{1}$.

(g) The function $t \mapsto \mathrm{E}\left(L,(1-t) \phi_{1}+t \phi_{2}, \phi_{3}\right)$ is a polynomial in $t \in[0,1]$ of degree $\leq n+1$.

(h) $\left.\frac{d}{d t}\right|_{t=0} \mathrm{E}\left(L,(1-t) \phi_{1}+t \phi_{2}, \phi_{1}\right)=\int_{X^{a n}}\left(\phi_{2}-\phi_{1}\right)\left(d d^{c} \phi_{1}\right)^{n}$.

(i) $\left.\frac{d^{2}}{d t^{2}}\right|_{t=0} \mathrm{E}\left(L,(1-t) \phi_{1}+t \phi_{2}, \phi_{1}\right)=n \int_{X^{a n}}\left(\phi_{2}-\phi_{1}\right) d d^{c}\left(\phi_{2}-\phi_{1}\right) \wedge\left(d d^{c} \phi_{1}\right)^{n-1}$.

(j) $\int_{X^{\text {an }}}\left(\phi_{1}-\phi_{2}\right)\left(d d^{c} \phi_{1}\right)^{n} \leq E\left(L, \phi_{1}, \phi_{2}\right) \leq \int_{X^{a n}}\left(\phi_{1}-\phi_{2}\right)\left(d d^{c} \phi_{2}\right)^{n}$.

(k) $\left|\mathrm{E}\left(L, \phi_{1}, \phi_{2}\right)-\mathrm{E}\left(L, \phi_{1}^{\prime}, \phi_{2}^{\prime}\right)\right| \leq\left(d\left(\phi_{1}, \phi_{1}^{\prime}\right)+d\left(\phi_{2}, \phi_{2}^{\prime}\right)\right) \operatorname{deg}_{L}(X)$.

(l) The energy is compatible with base extensions of non-Archimedean fields.

(m) If $f: X^{\prime} \rightarrow X$ is a birational proper morphism, then $\mathrm{E}\left(f^{*} L, f^{*} \phi_{1}, f^{*} \phi_{2}\right)=\mathrm{E}\left(L, \phi_{1}, \phi_{2}\right)$.

Proof. All these properties follow rather formally from the definition of the energy and the properties of the Monge-Ampère measures given in the previous subsection. For the arguments, we refer to [BFJ15, §6], [BJ18, $\S 3.8$ ] and [BE21, Proposition 9.14]. Note that for (f) and (j), we need the Hodge index result of Yuan and Zhang recalled in Proposition 2.26 .

\subsection{Non-Archimedean volumes}

In this subsection, we denote by $X$ a reduced proper scheme over $K$. We consider a line bundle $L$ on $X$ and set $N_{m}:=h^{0}(X, m L)$ for any $m \in \mathbb{N}$.

2.30. Let $V$ be an $N$-dimensional $K$-vector space. Then a norm $\|\cdot\|$ on $V$ induces a determinant norm $\operatorname{det}(\|\cdot\|)$ on the $\operatorname{determinant}$ line $\operatorname{det}(V)=\Lambda^{N}(V)$ by

$$
\operatorname{det}(\|\tau\|):=\inf _{\tau=v_{1} \wedge \cdots \wedge v_{N}}\left\|v_{1}\right\| \cdots\left\|v_{N}\right\|
$$

for any $\tau \in \operatorname{det}(V)$ (see [BE21, §2.1] for details). We define the relative volume of norms $\|\cdot\|_{1},\|\cdot\|_{2}$ on $V$ by

$$
\operatorname{vol}\left(\|\cdot\|_{1},\|\cdot\|_{2}\right):=\log \left(\frac{\operatorname{det}\left(\|\cdot\|_{2}\right)}{\operatorname{det}\left(\|\cdot\|_{1}\right)}\right)
$$

Note here that $\operatorname{det}\left(\|\cdot\|_{2}\right) / \operatorname{det}\left(\|\cdot\|_{1}\right)$ is a well-defined positive number since $\operatorname{det}(V)$ is a one-dimensional $K$-vector space. For more details on relative volumes, we refer to [BE21, §2.3].

Remark 2.31. Recall from [BE21, §1.7] that a lattice $\mathcal{V}$ in $V$ has an associated lattice norm $\|\cdot\|_{\mathcal{V}}$ on $V$ given by

$$
\|v\|_{\mathcal{V}}:=\inf _{\alpha \in K, v \in \alpha \mathcal{V}}|\alpha|
$$

for any $v \in V$. It follows from 2.5 that a finitely presented torsion $K^{\circ}$-module $M$ is given by $M=\mathcal{V}_{1} / \mathcal{V}_{2}$ for two lattices $\mathcal{V}_{1} \supset \mathcal{V}_{2}$ of a finite dimensional $K$-vector space $V$. Conversely, any such quotient is evidently a finitely presented torsion $K^{\circ}$-module. Then [BE21, Lemma 2.20] yields

$$
\mathrm{c}(M)=\operatorname{vol}\left(\|\cdot\|_{\mathcal{V}_{1}},\|\cdot\|_{\mathcal{V}_{2}}\right) \text {. }
$$

For any lattices $\mathcal{V}_{1}, \mathcal{V}_{2}$ in $V$, additivity of the relative volume [BE21, Proposition 2.14(i)] yields

$$
\mathrm{c}\left(\mathcal{V}_{1} / \mathcal{V}_{2}\right)=\operatorname{vol}\left(\|\cdot\|_{\mathcal{V}_{1}},\|\cdot\|_{\mathcal{V}_{2}}\right)
$$

where the content of the virtual $K^{\circ}$-module $\mathcal{V}_{1} / \mathcal{V}_{2}$ was defined in 2.9.

Definition 2.32. Let $\phi, \psi$ be bounded metrics on $L^{\text {an }}$. Then we define the non-Archimedean volume of $L$ with respect to $\phi$ and $\psi$ by

$$
\operatorname{vol}(L, \phi, \psi):=\limsup _{m \rightarrow \infty} \frac{n !}{m^{n+1}} \operatorname{vol}\left(\|\cdot\|_{m \phi},\|\cdot\|_{m \psi}\right)=\operatorname{vol}(L) \cdot \limsup _{m \rightarrow \infty} \frac{1}{m N_{m}} \operatorname{vol}\left(\|\cdot\|_{m \phi},\|\cdot\|_{m \psi}\right) .
$$

using the relative volume of the sup-norms $\|\cdot\|_{m \phi},\|\cdot\|_{m \psi}$ on $H^{0}(X, m L)$ from 2.17 . 
It follows from homogeneity and monotonicity of the relative volume of norms in [BE21, Proposition 2.14] that both limsup's are finite. By the right-hand equality, non-Archimedean volumes are thus interesting only when the line bundle $L$ is big.

We will see in Theorem 2.38 that the limsup in (2.4) is a limit if $X$ is geometrically reduced.

The non-Archimedean volume has the following basic properties holding for any proper reduced $X$. Recall that $d$ denotes the distance on the space of bounded metrics of a given line bundle (see 2.17).

Proposition 2.33. Let $\phi, \psi, \phi^{\prime}, \psi^{\prime}$ be bounded metrics on $L^{\text {an }}$. Then we have:

(a) $\operatorname{vol}(L, \phi, \phi)=0$.

(b) The non-Archimedean volume $\operatorname{vol}(L, \phi, \psi)$ is increasing in $\phi$ and decreasing in $\psi$.

(c) For any $c \in \mathbb{R}$, we have $\operatorname{vol}(L, \phi+c, \psi)=\operatorname{vol}(L, \phi, \psi)+c \operatorname{vol}(L)$.

(d) $\operatorname{vol}(L, \phi, \psi) \leq d(\phi, \psi) \operatorname{vol}(L)$.

(e) $\left|\operatorname{vol}(L, \phi, \psi)-\operatorname{vol}\left(L, \phi^{\prime}, \psi^{\prime}\right)\right| \leq\left(d\left(\phi, \phi^{\prime}\right)+d\left(\psi, \psi^{\prime}\right)\right) \operatorname{vol}(L)$.

Proof. Properties (a), (b) are obvious and (c), (e) follow from [BE21, Proposition 2.14(ii),(iv)]. Finally (d) is a consequence of (a) and (e).

Lemma 2.34. Let $\phi, \psi$ be bounded metrics on $L^{\text {an }}$. We suppose that either the valuation on $K$ is discrete or that $\phi, \psi$ are model metrics induced by line bundles on models which are integrally closed in $X$. Then

$$
c\left(\frac{\widehat{H^{0}}(X, m L, m \phi)}{\widehat{H^{0}}(X, m L, m \psi)}\right)=\operatorname{vol}\left(\|\cdot\|_{m \phi},\|\cdot\|_{m \psi}\right)+O\left(N_{m}\right) .
$$

Proof. By 2.18, we have that $B_{m}:=\widehat{H^{0}}\left(X, L^{\otimes m}, m \phi\right)$ and $B_{m}^{\prime}:=\widehat{H^{0}}\left(X, L^{\otimes m}, m \psi\right)$ are lattices in $H^{0}(X, m L)$. Let $\|\cdot\|_{B_{m}}$ and $\|\cdot\|_{B_{m}^{\prime}}$ be the associated lattice norms. Then we have

$$
\operatorname{vol}\left(\|\cdot\|_{B_{m}},\|\cdot\|_{B_{m}^{\prime}}\right)=\operatorname{vol}\left(\|\cdot\|_{m \phi},\|\cdot\|_{m \psi}\right)+O\left(N_{m}\right) .
$$

When $K$ is densely valued, (2.6) is actually an equality as then obviously $\|\cdot\|_{B_{m}}=\|\cdot\|_{m \phi}$ and $\|\cdot\|_{B_{m}^{\prime}}=\|\cdot\|_{m \psi}$. When $K$ is discretely valued, (2.6) holds by [BE21, Proposition 2.21]. Now the claim follows from (2.6) and Remark 2.31.

Remark 2.35. If the valuation on $K$ is discrete or if $\phi, \psi$ are model metrics induced by line bundles on models which are integrally closed in $X$, then Lemma 2.34 implies

$$
\operatorname{vol}(L, \phi, \psi)=\limsup _{m \rightarrow \infty} \frac{n !}{m^{n+1}} \mathrm{c}\left(\frac{\widehat{H^{0}}(X, m L, m \phi)}{\widehat{H^{0}}(X, m L, m \psi)}\right) .
$$

In the case of a discretely valued field with $v(\pi)=1$ for an uniformizer $\pi$, we conclude that $\operatorname{vol}(L, \phi, \psi)$ agrees with the non-Archimedean volume considered in $\left[\mathrm{BGJ}^{+} 20\right]$.

By Chow's lemma, the following result can be used to reduce to the case of projective schemes.

Lemma 2.36. Let $f: X^{\prime} \rightarrow X$ be a birational map of proper reduced schemes over $X$. For bounded metrics $\phi, \psi$ of the line bundle $L$ over $X$, we have

$$
\operatorname{vol}\left(\|\cdot\|_{m f^{*} \phi},\|\cdot\|_{m f^{*} \psi}\right)=\operatorname{vol}\left(\|\cdot\|_{m \phi},\|\cdot\|_{m \psi}\right)+o\left(m^{n+1}\right)
$$

for $m \rightarrow \infty$ and hence

$$
\operatorname{vol}\left(f^{*} L, f^{*} \phi, f^{*} \psi\right)=\operatorname{vol}(L, \phi, \psi) .
$$

Proof. This follows from [BE21, Lemma 9.11(v)] and its proof. Note that the projectivity assumption there can be replaced by properness. 
Next, we give the behaviour of the relative volumes in Definition 2.32 with respect to a base extension $F / K$ of non-Archimedean fields. We recall from [BE21, Definition 1.24, Proposition 1.25] that the base change of an ultrametric norm $\|\cdot\|$ on a $K$-vector space $V$ is the ultrametric norm on the $F$-vector space $V_{F}:=V \otimes_{K} F$ given for $w \in V_{F}$ by

$$
\|w\|_{F}:=\inf _{\sum \alpha_{i} v_{i}=w} \max _{i}\left|\alpha_{i}\left\|\mid v_{i}\right\|\right.
$$

where the infimum runs over all finite decompositions $\sum \alpha_{i} v_{i}=w$ with $\alpha_{i} \in F$ and $v_{i} \in V$. We will use similar notation to denote base changes of schemes and line bundles.

Proposition 2.37. We assume that $X$ is geometrically reduced. Let $\phi, \psi$ be continuous metrics on $L^{\text {an }}$ and let $F / K$ be a non-Archimedean field extension. Then

$$
\operatorname{vol}\left(\|\cdot\|_{m \phi},\|\cdot\|_{m \psi}\right)=\operatorname{vol}\left(\|\cdot\|_{m \phi_{F}},\|\cdot\|_{m \psi_{F}}\right)+o\left(m N_{m}\right)
$$

for $m \rightarrow \infty$ and hence $\operatorname{vol}\left(L_{F}, \phi_{F}, \psi_{F}\right)=\operatorname{vol}(L, \phi, \psi)$.

Proof. This follows from [BE21, Lemma 9.4]. Again, projectivity is not used there.

The next result is a consequence of the limit theorem of Chen and Maclean [CM15, Theorem 4.3] as shown in [BE21, §9.2].

Theorem 2.38. Let $L$ be a line bundle on a geometrically reduced proper scheme $X$ over $K$ and let $\phi, \psi$ be bounded metrics on $L^{\mathrm{an}}$. Then the limsup in the definition of the non-Archimedean volume $\operatorname{vol}(L, \phi, \psi)$ is a limit, i.e.

$$
\operatorname{vol}(L, \phi, \psi)=\lim _{m} \frac{n !}{m^{n+1}} \operatorname{vol}\left(\|\cdot\|_{m \phi},\|\cdot\|_{m \psi}\right) .
$$

The same holds in (2.7).

Proof. We reduce to $X$ projective by Lemma 2.36. Then the first claim follows from [BE21, Theorem 9.8]. The second claim follows from the first claim and (2.5).

The existence of the limit has the following obvious consequences. They simplify the proofs of the main results in this paper quite a lot, however one could also prove them without Theorem 2.38 and without Corollary 2.39 similarly as in $\left[\mathrm{BGJ}^{+} 20\right]$.

Corollary 2.39. Let $L$ be a line bundle on an $n$-dimensional geometrically reduced proper scheme $X$ over $K$ and let $\phi, \psi, \phi_{1}, \phi_{2}, \phi_{3}$ be bounded metrics on $L^{\mathrm{an}}$. Then we have:

(i) $\operatorname{vol}(L, \phi, \psi)=-\operatorname{vol}(L, \psi, \phi)$

(ii) $\operatorname{vol}\left(L, \phi_{1}, \phi_{2}\right)+\operatorname{vol}\left(L, \phi_{2}, \phi_{3}\right)+\operatorname{vol}\left(L, \phi_{3}, \phi_{1}\right)=0$.

(iii) $\operatorname{vol}(a L, a \phi, a \psi)=a^{n+1} \operatorname{vol}(L, \phi, \psi)$ for all $a \in \mathbb{N}$.

\section{Volumes on torsion schemes}

Let $K$ be any non-Archimedean field, with valuation $v$. In this section, we consider schemes over $K^{\circ}$ which have non-trivial $K^{\circ}$-torsion. We call them torsion schemes over $K^{\circ}$. Our main examples are closed subschemes of a scheme over $K^{\circ}$ with support in the special fiber. We will see that the space of global sections of a line bundle $L$ over a finitely presented projective torsion scheme over $K^{\circ}$ is a finitely presented torsion $K^{\circ}$-module and hence we can define the volume of $L$ by mimicking the classical construction from algebraic geometry by using the content from $\$ 2.2$ instead of the dimension. We will prove some basic properties of the volume analogously to $\left[\mathrm{BGJ}^{+} 20\right.$, Section 3]. The main difficulty here is that our torsion schemes are not Noetherian unless the valuation is discrete. 


\subsection{Hilbert-Samuel theory}

We introduce torsion schemes over $K^{\circ}$, define the numbers $h^{q}(Y, \mathcal{F})$ for a coherent sheaf $\mathcal{F}$ over such a torsion scheme $Y$, and show that they fit in the usual Hilbert-Samuel theory.

Definition 3.1. We say that a $K^{\circ}$-scheme $Y$ is a torsion scheme over $K^{\circ}$ if $A$ is a torsion $K^{\circ}$-module for any open affine subset $\operatorname{Spec}(A)$ of $Y$.

Remark 3.2. Now assume that $Y$ is a torsion scheme of finite type over $K^{\circ}$. Then for any open affine subset $\operatorname{Spec}(A)$ of $Y$ there is a non-zero element $a \in K^{\circ}$ such that $a \cdot A=0$. Moreover, $Y$ is a torsion scheme over $K^{\circ}$ if and only if there exists some non zero $b \in K^{\circ}$, some scheme $Y^{\prime}$ over Spec $K^{\circ} /(b)$ such that $Y^{\prime}$ is isomorphic to the $Y$ as a scheme over $\operatorname{Spec} K^{\circ}$. If $Y$ is projective (resp. proper), then $Y^{\prime}$ is projective (resp. proper) as well.

3.3. Let $\alpha \in K^{\circ}$ and let $A:=K^{\circ} /(\alpha)$. We pick an $n \in \mathbb{N}$ and set $S:=A\left[x_{0}, \ldots, x_{n}\right]$. We consider the standard $\mathbb{N}$-grading on $S$.

Definition 3.4. Let $n \in \mathbb{N}$ and $M$ be an $S$-graded module of finite presentation. We denote by $M_{j}$ the elements of $M$ of degree $j \in \mathbb{N}$. Note that $M_{j}$ is a finitely presented torsion $K^{\circ}$-module and hence we may use the content from $\S 2.2$ to define the Hilbert function of $M$ by

$$
P_{M}(j):=\mathrm{c}\left(M_{j}\right) \text {. }
$$

Lemma 3.5. Let $0 \rightarrow M^{\prime} \rightarrow M \rightarrow M^{\prime \prime} \rightarrow 0$ be a short exact sequence of finitely presented graded $S$-modules. Then we have $P_{M^{\prime}}+P_{M^{\prime \prime}}=P_{M}$.

Proof. This follows easily from Proposition 2.8 .

Lemma 3.6. Let $\psi: M \rightarrow N$ be a morphism of finitely presented $S$-modules. Then $\operatorname{Ker}(\psi)$ and $\operatorname{Coker}(\psi)$ are finitely presented $S$-modules.

Proof. It follows from [Ul195, Example 3.3] that the ring $A$ is stably (universally) coherent and hence the lemma follows from standard properties of modules over coherent rings (see for instance [Stacks, Tags 05CX and $05 \mathrm{CW}])$.

Remark 3.7. Ullrich's results [Ul195, Example 3.3] show that every finitely presented $K^{\circ}$-algebra is coherent, and hence that the structure sheaf of any finitely presented scheme $Y$ over $K^{\circ}$ is coherent. As a result, an $\mathcal{O}_{Y}$-module is coherent if and only if it is finitely presented. This yields that the pull-back of a coherent module with respect to a morphism of finitely presented (torsion) schemes over $K^{\circ}$ is again coherent (see [Gro60, §0.5.3]). The direct image theorem [Ul195, Theorem 3.5] with respect to a proper morphism of finitely presented schemes over $K^{\circ}$ holds. In particular, for a coherent sheaf $\mathcal{F}$ on a finitely presented proper scheme $Y$ over $K^{\circ}$, all cohomology groups $H^{i}(Y, \mathcal{F})$ are finitely presented $K^{\circ}$-torsion modules.

Definition 3.8. Let $\mathcal{F}$ be a coherent sheaf on a finitely presented proper torsion scheme over $K^{\circ}$. Then we define

$$
h^{q}(Y, \mathcal{F}):=\mathrm{c}\left(H^{q}(Y, \mathcal{F})\right)
$$

for any $q \in \mathbb{N}$ and the Euler characteristic

$$
\chi(Y, \mathcal{F}):=\sum_{q=0}^{\operatorname{dim}(Y)}(-1)^{q} h^{q}(Y, \mathcal{F}) .
$$

Proposition 3.9. Let $Y$ be a finitely presented projective torsion scheme over $K^{\circ}$ and let $L_{1}, \ldots, L_{r}$ be line bundles on $Y$. Then for any coherent sheaf $\mathcal{F}$ on $Y$, the Euler characteristic $\chi\left(Y, \mathcal{F}\left(m_{1} L_{1}+\cdots+m_{r} L_{r}\right)\right)$ is a polynomial function of $\left(m_{1}, \ldots, m_{r}\right) \in \mathbb{Z}^{r}$. 
Proof. In a first step, we assume that $L_{1}, \ldots, L_{r}$ are very ample, and follow the lines of the classical proof. We have a closed embedding $i: Y \rightarrow \mathbb{P}_{K^{\circ}}^{n_{1}} \times \cdots \times \mathbb{P}_{K^{\circ}}^{n_{r}}$ with $L_{i} \cong i^{*} \mathcal{O}\left(e_{i}\right)$ and using that cohomology does not change after passing to the push-forward $i_{*}$, we may assume that $Y=\mathbb{P}_{K^{\circ}}^{n_{1}} \times \cdots \times \mathbb{P}_{K^{\circ}}^{n_{r}}$ and $L_{i}=\mathcal{O}\left(e_{i}\right)$ for $i=1, \ldots, r$ where $e_{1}, \ldots, e_{r}$ is the standard basis of $\mathbb{Z}^{r}$. The homogeneous coordinates on $\mathbb{P}_{K^{\circ}}^{n_{i}}$ are denoted by $y_{i 0}, \ldots, y_{i n_{i}}$. We proceed by induction on $N:=\sum_{i=1}^{r} n_{i}$.

If $N=0$, then $Y=\mathbb{P}_{K^{\circ}}^{0}$ and all line bundles $L_{i}$ are trivial, hence $\chi\left(Y, \mathcal{F}\left(m_{1} L_{1}+\cdots+m_{r} L_{r}\right)\right)$ is a constant function. So we may assume $N>0$ and that the claim holds for $N-1$. We may assume that $n_{i}>0$ for all $i=0, \ldots, r$. Then we consider the morphism $\psi: \mathcal{F}\left(-e_{i}\right) \rightarrow \mathcal{F}$ induced by multiplication with $y_{i n_{i}}$ and the induced short exact sequence

$$
0 \rightarrow \operatorname{Ker}(\psi) \rightarrow \mathcal{F}\left(-e_{i}\right) \rightarrow \mathcal{F} \rightarrow \operatorname{Coker}(\psi) \rightarrow 0 .
$$

It is clear that $\operatorname{Ker}(\psi)$ and $\operatorname{Coker}(\psi)$ are coherent sheaves on $Y=\mathbb{P}_{K^{\circ}}^{n_{1}} \times \cdots \times \mathbb{P}_{K^{\circ}}^{n_{r}}$. In fact, the definition of $\psi$ as multiplication by $y_{i n_{i}}$ yields easily that they are defined on the closed subscheme $y_{i n_{i}}=0$ which is isomorphic to $\mathbb{P}_{K^{\circ}}^{n_{1}} \times \cdots \times \mathbb{P}_{K^{\circ}}^{n_{i}-1} \times \cdots \times \mathbb{P}_{K^{\circ}}^{n_{r}}$. By induction hypothesis, we now get the claim for the coherent sheaves $\operatorname{Ker}(\psi)$ and $\operatorname{Coker}(\psi)$. We twist the above exact sequence with $m_{1} L_{1}+\cdots+m_{r} L_{r}$ and then we apply additivity of the Euler characteristic to the resulting exact sequence to deduce that

$$
\chi\left(Y, \mathcal{F}\left(m_{1} L_{1}+\cdots+m_{r} L_{r}\right)\right)-\chi\left(Y, \mathcal{F}\left(m_{1} L_{1}+\cdots+m_{r} L_{r}-L_{i}\right)\right)
$$

is equal to

$$
\chi\left(Y, \operatorname{Coker}(\psi)\left(m_{1} L_{1}+\cdots+m_{r} L_{r}\right)\right)-\chi\left(Y, \operatorname{Ker}(\psi)\left(m_{1} L_{1}+\cdots+m_{r} L_{r}\right)\right)
$$

and hence it is a polynomial function. Now we use the following fact for any function $f: \mathbb{Z}^{r} \rightarrow \mathbb{R}$. If we know that $f(x)-f\left(x-e_{i}\right)$ is a polynomial function for all $i=1, \ldots, r$, then it is quite easy to see that $f(x)$ is a polynomial function. This implies that $\chi\left(Y, \mathcal{F}\left(m_{1} L_{1}+\cdots+m_{r} L_{r}\right)\right)$ is a polynomial function in $\left(m_{1}, \ldots, m_{r}\right)$, proving the first step.

We now consider the general case. By [GW10, Theorem 13.59], we can find very ample line bundles $A_{i}, B_{i}$ such that $L_{i}=A_{i}-B_{i}$. The first step shows that

$$
\chi\left(Y, \mathcal{F}\left(p_{1} A_{1}+q_{1} B_{1}+\cdots+p_{r} A_{r}+q_{r} B_{r}\right)\right)
$$

is a polynomial function in $\left(p_{1}, q_{1}, \ldots, p_{r}, q_{r}\right) \in \mathbb{Z}^{2 r}$. Applying this with $p_{i}=m_{i}$ and $q_{i}=-m_{i}$ for all $i=1, \ldots, r$, we get the claim.

Lemma 3.10. Let $Y$ be a finitely presented projective torsion scheme over $K^{\circ}$ and let $L_{1}, \ldots, L_{r}$ be ample line bundles on $Y$. Then for any coherent sheaf $\mathcal{F}$ on $Y$, we have

$$
H^{q}\left(Y, \mathcal{F}\left(m_{1} L_{1}+\cdots+m_{r} L_{r}\right)\right)=0
$$

for all $m_{1}, \ldots, m_{r} \in \mathbb{N}$ with $m_{1}+\cdots+m_{r}$ sufficiently large and all $q>0$.

Proof. For $r=1$, this is Serre's vanishing theorem, $c f$. [Ull95, Proposition 3.6]. We prove now the claim for $r \geq 2$. We note that there is $k \in \mathbb{N} \backslash\{0\}$ such that $H_{j}:=k L_{j}$ is very ample for every $j=1, \ldots, r$. Writing $m_{j}=m_{j}^{\prime} k+d_{j}$ for $m_{j}^{\prime} \in \mathbb{N}$ and $d_{j} \in\{0, \ldots, k-1\}$, we see that it is enough to prove the claim for the finitely many coherent sheaves $\mathcal{F}\left(d_{1} L_{1}+\cdots+d_{r} L_{r}\right)$ and the very ample line bundles $H_{j}$. We conclude that we may assume all $L_{j}$ very ample.

There is a suitable closed immersion into a multiprojective space $\mathbb{P}=\mathbb{P}_{K^{\circ}}^{n_{1}} \times \cdots \times \mathbb{P}_{K^{\circ}}^{n_{r}}$ over $K^{\circ}$ such that $L_{j}=\left.\mathcal{O}_{\mathbb{P}}\left(e_{j}\right)\right|_{Y}$ for $j=1, \ldots, r$, where $\mathcal{O}_{\mathbb{P}}\left(e_{j}\right)$ is the pull-back of $\mathcal{O}_{\mathbb{P}_{K^{\circ}}^{n_{j}}}(1)$ with respect to the $j$-th projection. We choose such an embedding with $\mathbb{P}$ of minimal dimension. The proof will run by induction over $\operatorname{dim}(\mathbb{P})$. If $\operatorname{dim}(\mathbb{P})=0$, then $\operatorname{dim}(Y)=0$ and the claim is obvious.

Now we assume $\operatorname{dim}(\mathbb{P}) \geq 1$. For every $i \in\{1, \ldots, r\}$, we pick a homogeneous coordinate $x_{i}$ on the factor $\mathbb{P}_{K^{\circ}}^{n_{i}}$ and set $\mathbb{P}_{i}:=\left\{x \in \mathbb{P} \mid x_{i}=0\right\}$ for the associated multiprojective space with $\operatorname{dim}\left(\mathbb{P}^{\prime}\right)=\operatorname{dim}(\mathbb{P})-1$. Then $Y_{i}:=Y \times_{\mathbb{P}} \mathbb{P}_{i}$ is a finitely presented torsion scheme over $K^{\circ}$ which is a closed subscheme of $Y$ and of $\mathbb{P}_{i}$. 
The restriction $\mathcal{F}_{i}$ of $\mathcal{F}$ to $Y^{\prime}$ is a coherent sheaf on $Y^{\prime}$ and the restriction of $L_{j}$ to $Y_{i}$ is a very ample line bundle $L_{i j}$ for $j=1, \ldots, r$. By induction and using that the $\ell^{1}$-norm of $\left(m_{i}\right)$ and its $\ell^{\infty}$-norm are equivalent, there is $\ell \in \mathbb{N}$ such that

$$
H^{q}\left(Y_{i}, \mathcal{F}_{i}\left(m_{1} L_{i 1}+\cdots+m_{r} L_{i r}\right)\right)=0
$$

for all $m_{1}, \ldots, m_{r} \in \mathbb{N}$ with $\max \left\{m_{1}, \ldots, m_{r}\right\} \geq \ell$ and all $i \in\{1, \ldots, r\}$. Using the case $r=1$, we may choose $\ell$ so large such that $H^{q}\left(Y, \mathcal{F}\left(m_{j} L_{j}\right)\right)=0$ for all $m_{j} \geq \ell$ and all $j=1, \ldots, r$. We claim that for all $m_{1}, \ldots, m_{r} \in \mathbb{N}$ with $\max \left\{m_{1}, \ldots, m_{r}\right\} \geq \ell$ we have

$$
H^{q}\left(Y, \mathcal{F}\left(m_{1} L_{1}+\cdots+m_{r} L_{r}\right)\right)=0
$$

which implies the lemma. We argue by contradiction. We choose $m_{1}, \ldots, m_{r}$ minimal with respect to the product (partial) order on $\mathbb{N}^{r}$ such that (3.2) is wrong. Let $m_{j}$ be the maximal $m_{1}, \ldots, m_{r}$. There is $i \neq j$ such that $m_{i}>0$, otherwise (3.2) would be true by the choice of $\ell$ using the case $r=1$. Since $x_{i}$ is a regular global section of $\mathcal{O}_{\mathbb{P}}\left(e_{i}\right)$ (see the paragraph before Proposition 3.18 for the definition of regular), the sequence

$$
0 \longrightarrow \mathcal{O}_{\mathbb{P}}\left(-e_{i}\right) \stackrel{\otimes x_{i}}{\longrightarrow} \mathcal{O}_{\mathbb{P}} \longrightarrow \mathcal{O}_{\mathbb{P}_{i}} \longrightarrow 0
$$

on $\mathbb{P}$ is exact. Then the associated long exact cohomology sequence is

$$
\cdots \longrightarrow \underbrace{H^{q}\left(\mathbb{P}, \mathcal{F}\left(m e-e_{i}\right)\right)}_{H^{q}\left(Y, \mathcal{F}\left(m L-L_{i}\right)\right)} \longrightarrow \underbrace{H^{q}(\mathbb{P}, \mathcal{F}(m e))}_{H^{q}(Y, \mathcal{F}(m L))} \longrightarrow \underbrace{H^{q}\left(\mathbb{P}_{i}, \mathcal{F}(m e)\right)}_{H^{q}\left(Y_{i}, \mathcal{F}_{i}\left(m L_{i}\right)\right)} \longrightarrow \cdots
$$

where $m e:=m_{1} e_{1}+\cdots+m_{r} e_{r}, m L:=m_{1} L_{1}+\cdots+m_{r} L_{r}$ and $m L_{i}=m_{1} L_{i 1}+\cdots+m_{r} L_{i r}$. By (3.1), we have $H^{q}\left(Y_{i}, \mathcal{F}_{i}\left(m L_{i}\right)\right)=0$. Using that $\max \left\{m_{1}, \ldots, m_{r}\right\}$ does not change if we replace $m_{i}$ by $m_{i}-1$, minimality yields that $H^{q}\left(Y, \mathcal{F}\left(m L-L_{i}\right)\right)=0$ and hence $H^{q}(Y, \mathcal{F}(m L))=0$. This is a contradiction and the lemma follows.

Corollary 3.11. Let $L_{1}, \ldots, L_{r}$ be ample line bundles on a finitely presented projective torsion scheme $Y$ over $K^{\circ}$. Then for any coherent sheaf $\mathcal{F}$ on $Y$, we have that $h^{0}\left(Y, \mathcal{F}\left(m_{1} L_{1}+\cdots+m_{r} L_{r}\right)\right)$ is a polynomial function of $\left(m_{1}, \ldots, m_{r}\right) \in \mathbb{N}^{r}$ for $m_{1}+\cdots+m_{r}$ large enough.

Proof. This follows from Proposition 3.9 and Lemma 3.10.

\subsection{Global sections in the non-Noetherian case}

The goal of this subsection is to prove that a line bundle on a finitely presented projective torsion $K^{\circ}$-scheme can be written as a difference of effective line bundles which have global sections not vanishing on a given finite set of points of $Y$. This fact is established in Corollary 3.17.

3.12. Let $Y$ be a finitely presented torsion $K^{\circ}$-scheme. We denote by

$$
Y_{s}:=Y \times_{\operatorname{Spec} K} \circ \operatorname{Spec} \tilde{K}
$$

the special fiber of $Y$. We remark that the induced closed immersion $Y_{S} \rightarrow Y$ induces a homeomorphism of the underlying topological spaces $\left|Y_{s}\right| \simeq|Y|$.

Lemma 3.13. Let $Y$ be a torsion scheme of finite type over $K^{\circ}$. Let $\mathcal{F}$ be a sheaf on $Y$ and let $s \in H^{0}\left(Y_{s}, \mathcal{F}\right)$. Then there is some $r \in \mathbb{R}$ with $0<r<1$ such that for any $\beta \in K^{\circ}$ with $r \leq|\beta|<1$ the section $s$ can be lifted to $H^{0}\left(Y_{K^{\circ} /(\beta)}, \mathcal{F}\right)$ via the canonical morphism $H^{0}\left(Y_{K^{\circ} /(\beta)}, \mathcal{F}\right) \rightarrow H^{0}\left(Y_{s}, \mathcal{F}\right)$.

Proof. The isomorphism $\tilde{K} \simeq \lim _{\longrightarrow} K^{\circ} /(\beta)$, where $\beta \in K^{\circ \circ}$, induces an isomorphism

$$
\mathcal{O}_{Y_{s}} \simeq \underset{\beta \in K^{\circ \circ}}{\lim } \mathcal{O}_{Y_{K^{\circ} /(\beta)}}
$$


of abelian sheaves on the topological space $|Y|$. Let us denote by $\mathcal{F}_{Y_{K^{\circ} /(\beta)}}$ (resp. $\mathcal{F}_{Y_{s}}$ ) the pull-back of $\mathcal{F}$ to $Y_{K \circ /(\beta)}\left(\right.$ resp. $\left.Y_{s}\right)$. Then we get similarly

$$
\mathcal{F}_{Y_{s}} \simeq \underset{\beta \in K^{\circ \circ}}{\lim } \mathcal{F}_{Y_{K^{\circ} /(\beta)}}
$$

By [Har77, Chapter II, Exercise 1.11], we get that

$$
H^{0}\left(Y, \mathcal{F}_{Y_{s}}\right) \simeq \underset{\beta}{\lim } H^{0}\left(Y_{K^{\circ} /(\beta)}, \mathcal{F}_{Y_{K^{\circ} /(\beta)}}\right)
$$

which proves the above result.

Lemma 3.14. Let $Y$ be a projective torsion scheme over $K^{\circ}$ with a coherent $\mathcal{O}_{Y}$-module $\mathcal{F}$ and an ample line bundle $A$. Then there is some $r \in \mathbb{R}$ with $0<r<1$ such that for any $\beta \in K^{\circ}$ with $r \leq|\beta|<1$ and for any $m \in \mathbb{N}$, the morphism

$$
H^{0}\left(Y_{K \circ /(\beta)}, \mathcal{F}(m A)\right) \rightarrow H^{0}\left(Y_{s}, \mathcal{F}(m A)\right)
$$

is surjective.

Proof. We consider the ring

$$
R:=\bigoplus_{m \in \mathbb{N}} H^{0}\left(Y_{s}, m A\right)
$$

and the $R$-module

$$
M:=\bigoplus_{m \in \mathbb{N}} H^{0}\left(Y_{s}, \mathcal{F}(m A)\right)
$$

Since $A$ is ample on the projective scheme $Y_{S}$ over the residue field $\tilde{K}$, it follows that $R$ is a $\tilde{K}$-algebra of finite type and that $M$ is a finitely generated $R$-module [Laz04a, Example 1.2.22].

Then we first pick a finite set $\left\{r_{i}\right\}_{i \in I}$ of generators of the $\tilde{K}$-algebra $R$. For $\beta \in K^{\circ \circ}$ with $|\beta|<1$ close enough to 1, Lemma 3.13 shows that we can lift all the $r_{i}$ to

$$
R_{\beta}:=\bigoplus_{m \in \mathbb{N}} H^{0}\left(Y_{K^{\circ} /(\beta)}, m A\right)
$$

Similarly we pick a finite set $\left\{m_{j}\right\}_{j \in J}$ of generators of the finite $R$-module $M$. For $\beta \in K^{\circ \circ}$ with $|\beta|<1$ close enough to 1 , Lemma 3.13 again shows that all the $m_{j}$ lift to

$$
M_{\beta}:=\bigoplus_{m \in \mathbb{N}} H^{0}\left(Y_{K^{\circ} /(\beta)}, \mathcal{F}(m A)\right)
$$

This proves that for $\beta \in K^{\circ \circ}$ with $|\beta|<1$ close enough to 1 such that the above liftings are possible, the morphism $M_{\beta} \rightarrow M$ is surjective. Clearly, this proves the claim.

Lemma 3.15. Let $Y$ be a finitely presented projective torsion scheme over $K^{\circ}$. Let $\mathcal{F}$ be a coherent $\mathcal{O}_{Y}$-module and let $A$ be an ample line bundle on $Y$. Then there exists some $M \in \mathbb{N}$ such that for any integer $m \geq M$, the morphism $H^{0}(Y, \mathcal{F}(m A)) \rightarrow H^{0}\left(Y_{s}, \mathcal{F}(m A)\right)$ is surjective.

Proof. First choose some non-zero $\alpha \in K^{\circ \circ}$ such that $Y$ is defined over $K^{\circ} /(\alpha)$. By Lemma 3.14, there is some $r \in \mathbb{R}$ with $|\alpha|<r<1$ such that for any $\beta \in K^{\circ}$ with $r \leq|\beta|<1$ and for any $m \in \mathbb{N}$, the morphism

$$
H^{0}\left(Y_{K^{\circ} /(\beta)}, \mathcal{F}(m A)\right) \rightarrow H^{0}\left(Y_{s}, \mathcal{F}(m A)\right)
$$

is surjective. If we tensor the surjective homomorphism $\mathcal{O}_{Y} \rightarrow \mathcal{O}_{Y_{K^{\circ} /(\beta)}}$ of coherent $\mathcal{O}_{Y}$-modules with $\mathcal{F}$, then we get an exact sequence

$$
0 \rightarrow \mathscr{M} \rightarrow \mathcal{F} \rightarrow \mathcal{O}_{Y_{K^{\circ} /(\beta)}} \otimes \mathcal{F} \rightarrow 0
$$

of coherent $\mathcal{O}_{Y}$-modules. Since $A$ is ample, it follows from [Ul195, Proposition 3.6] that

$$
0 \rightarrow H^{0}(Y, \mathscr{M}(m A)) \rightarrow H^{0}(Y, \mathcal{F}(m A)) \rightarrow H^{0}\left(Y, \mathcal{O}_{Y_{K^{\circ} /(\beta)}} \otimes \mathcal{F}(m A)\right) \rightarrow 0 .
$$


is exact for $m \gg 0$. Since

$$
H^{0}\left(Y, \mathcal{O}_{Y_{K^{\circ} /(\beta)}} \otimes \mathcal{F}(m A)\right) \simeq H^{0}\left(Y_{K^{\circ} /(\beta)}, \mathcal{F}(m A)\right)
$$

we get the result by combining (3.3) and (3.4).

Lemma 3.16. Let $T$ be a finite subset of a finitely presented projective torsion scheme $Y$ over $K^{\circ}$. Let $\mathcal{F}$ be a line bundle on $Y$ and let $A$ be an ample line bundle on $Y$. Then there is $M \geq 0$ such that for any integer $m \geq M$ there exists a section $s \in H^{0}(Y, \mathcal{F}(m A))$ such that $s(t) \in \mathcal{F}_{t} / \mathrm{m}_{t} \mathcal{F}_{t}$ is non-zero for any $t \in T$ where $\mathfrak{m}_{t}$ is the maximal ideal in $\mathcal{O}_{Y, t}$.

Proof. Using that $Y_{S}$ is a projective scheme over the residue field $\tilde{K},\left[\mathrm{BGJ}^{+} 20\right.$, Lemma 3.1.1] yields that there is $M \geq 0$ such that for any integer $m \geq M$ there is $s \in H^{0}\left(Y_{s}, \mathcal{F}(m A)\right)$ which does not vanish at any point of $T$. Then the claim follows from Lemma 3.15.

Corollary 3.17. Let $Y$ be a finitely presented projective torsion scheme over $K^{\circ}$. Let $T \subset Y$ be finite and let $L$ be a line bundle on $Y$. Then there are very ample line bundles $A_{1}, A_{2}$ on $Y$ with $L \simeq A_{1}-A_{2}$ such that the line bundles $A_{i}$ have global sections $s_{i}$ with $s_{i}(t) \neq 0$ for any $t \in T$.

Proof. We pick an ample line bundle $A$ on $Y$. We apply Lemma 3.16 first with $\mathcal{F}=L$. For $m \gg 0$, we get that $m A+L$ has a global section $s_{1}$ not vanishing at any point of $T$. Then we apply Lemma 3.16 with $\mathcal{F}=\mathcal{O}_{Y}$. For $m \gg 0$, we get that $m A$ has a global section $s_{2}$ not vanishing at any point of $T$. Hence for $m \gg 0$, $A_{1}:=m A+L$ and $A_{2}:=m A$, we get the result. Note that for $m \gg 0$ these line bundles are very ample by [GW10, Theorem 13.59].

Any global section $s$ of a line bundle $L$ over a scheme $X$ defines a closed subscheme $D$ of $X$. We call $s$ regular if $D$ is a Cartier divisor of $X$. We will use the following relative version in case of a flat morphism $\pi: X \rightarrow Y$ of schemes. Then $s$ is called relatively regular if $D$ is a Cartier divisor of $X$ and if $D$ is flat over $Y$. We recall the following result from [BE21, Proposition A.15].

Proposition 3.18 (Boucksom-Eriksson). Let $\pi: X \rightarrow Y$ be a flat (finitely presented) projective morphism of schemes of finite presentation over $K^{\circ}$ and let $L$ be a $\pi$-ample line bundle on $X$. Then $m L$ has a relatively regular section locally over $Y$ for all $m \gg 0$.

\subsection{Asymptotics}

In this subsection, we will study the asymptotics of cohomology groups to introduce the volume of a line bundle over a finitely presented projective torsion scheme $Y$ over $K^{\circ}$. We will use the previous subsection to prove that the volume increases after adding an effective line bundle. We will explain why the non-Noetherian situation makes this surprisingly hard to prove. We start with a crucial continuity result.

Lemma 3.19. Let $\mathcal{F}$ be a coherent sheaf and let $L_{0}, \ldots, L_{r}$ be line bundles on $Y$. We set $n:=\operatorname{dim}(\operatorname{supp}(\mathcal{F}))$. Then for all $m_{1}, \ldots, m_{r} \in \mathbb{N} \backslash\{0\}$ and $m:=\sum_{i} m_{i}$, we have:

$$
\begin{gathered}
h^{q}\left(Y, \mathcal{F}\left(m_{1} L_{1}+\cdots+m_{r} L_{r}\right)\right)=O\left(m^{n}\right) \\
\left|h^{q}\left(Y, \mathcal{F}\left(L_{0}+m_{1} L_{1}+\cdots+m_{r} L_{r}\right)\right)-h^{q}\left(Y, \mathcal{F}\left(m_{1} L_{1}+\cdots+m_{r} L_{r}\right)\right)\right|=O\left(m^{n-1}\right)
\end{gathered}
$$

Proof. We note that the support of a coherent sheaf is closed. We use the shorthand notation

$$
\mathbf{m}:=\left(m_{1}, \ldots, m_{r}\right), \mathcal{F}(\mathbf{m}):=\mathcal{F}\left(m_{1} L_{1}+\cdots+m_{r} L_{r}\right) \text { and } \mathcal{F}\left(\mathbf{m}, L_{0}\right):=\mathcal{F}(\mathbf{m}) \otimes L_{0} .
$$

We prove both claims simultaneously by induction on $n:=\operatorname{dim}(\operatorname{supp}(\mathcal{F}))$. If the support is empty, then $\mathcal{F}=0$ and the claims are obvious as the left hand sides are zero.

Now we suppose that $n \geq 0$ and that the claims are known for all coherent sheaves whose support have dimension $<n$. 
By Corollary 3.17, there are line bundles $A, B$ with global sections $s_{A}, s_{B}$ such that $L_{0}=A-B$ and the supports of $s_{A}$ and $s_{B}$ both do not contain any generic point of $\operatorname{supp}(\mathcal{F})$. Let $E$ (resp. $F$ ) be the closed subscheme of $X$ defined by $s_{A}$ (resp. $\left.s_{B}\right)$. We conclude that the supports of $\left.\mathcal{F}(\mathbf{m})\right|_{E}$ and of $\left.\mathcal{F}(\mathbf{m}, A)\right|_{E}$ have dimension at most $n-1$ and the same also holds for the restrictions to $F$. We get an exact sequence

$$
\left.0 \longrightarrow \mathcal{G} \longrightarrow \mathcal{F} \stackrel{\otimes s_{A}}{\longrightarrow} \mathcal{F}(A) \longrightarrow \mathcal{F}(A)\right|_{E} \longrightarrow 0
$$

of coherent sheaves on $Y$. We twist by $m_{1} L_{1}+\cdots+m_{r} L_{r}$ and get the exact sequence

$$
\left.0 \longrightarrow \mathcal{G}(\mathbf{m}) \longrightarrow \mathcal{F}(\mathbf{m}) \stackrel{\otimes s_{A}}{\longrightarrow} \mathcal{F}(\mathbf{m}, A) \longrightarrow \mathcal{F}(\mathbf{m}, A)\right|_{E} \longrightarrow 0 .
$$

By the choice of the global section $s_{A}$, the dimension of $\operatorname{supp}(\mathcal{G})$ is at most $n-1$. By induction on $n$, we have

$$
h^{q}(Y, \mathcal{G}(\mathbf{m}))=O\left(m^{n-1}\right) \quad \text { and } \quad h^{q}\left(E,\left.\mathcal{F}(\mathbf{m}, A)\right|_{E}\right)=O\left(m^{n-1}\right)
$$

We split the exact sequence (3.7) into two short exact sequences

$$
0 \longrightarrow \mathcal{G} \longrightarrow \mathcal{F} \longrightarrow \mathcal{H} \longrightarrow 0 \text { and }\left.0 \longrightarrow \mathcal{H} \longrightarrow \mathcal{F}(A) \longrightarrow \mathcal{F}(A)\right|_{E} \longrightarrow 0
$$

We twist again these two short exact sequences by $m_{1} L_{1}+\cdots+m_{r} L_{r}$ and then we use the associated long exact cohomology sequences to deduce

$$
-h^{q-1}\left(E,\left.\mathcal{F}(\mathbf{m}, A)\right|_{E}\right) \leq h^{q}(Y, \mathcal{F}(\mathbf{m}, A))-h^{q}(Y, \mathcal{H}(\mathbf{m})) \leq h^{q}\left(E,\left.\mathcal{F}(\mathbf{m}, A)\right|_{E}\right)
$$

and

$$
-h^{q}(Y, \mathcal{G}(\mathbf{m})) \leq h^{q}(Y, \mathcal{H}(\mathbf{m}))-h^{q}(Y, \mathcal{F}(\mathbf{m})) \leq h^{q+1}(Y, \mathcal{G}(\mathbf{m}))
$$

Using these inequalities and (3.9), we get

$$
h^{q}(Y, \mathcal{F}(\mathbf{m}, A))-h^{q}(Y, \mathcal{F}(\mathbf{m}))=O\left(m^{n-1}\right) .
$$

We apply (3.11) to $\mathcal{F}^{\prime}:=\mathcal{F}(A-B)$ instead of $\mathcal{F}$ and $B$ instead of $A$ to get

$$
h^{q}\left(Y, \mathcal{F}^{\prime}(\mathbf{m}, B)\right)-h^{q}\left(Y, \mathcal{F}^{\prime}(\mathbf{m})\right)=O\left(m^{n-1}\right) .
$$

Using that $\mathcal{F}^{\prime}(\mathbf{m}) \simeq \mathcal{F}\left(\mathbf{m}, L_{0}\right)$ and that $\mathcal{F}^{\prime}(\mathbf{m}, B) \simeq \mathcal{F}(\mathbf{m}, A)$, the inequality (3.6) for $n$ follows easily from (3.11) and (3.12). It is clear that (3.5) follows from a repeated application of (3.6) by choosing $L_{0}$ from $L_{1}, \ldots, L_{r}$.

\subsection{Asymptotic cohomological functions on the real Picard group}

This subsection is inspired by the results about $\mathbb{R}$-divisors on reduced projective schemes over a field from $\left[\mathrm{BGJ}^{+} 20, \S 3\right]$.

Theorem 3.20. Let $Y$ be a finitely presented projective torsion scheme over $K^{\circ}$, of dimension $n$. For each $q=0, \ldots, n$, there exists a unique function $\hat{h}^{q}(Y, \cdot): \operatorname{Pic}(Y)_{\mathbb{R}} \rightarrow \mathbb{R}_{\geq 0}$ such that:

(i) for any $L \in \operatorname{Pic}(Y)$, we have

$$
\hat{h}^{q}(Y, L)=\limsup _{m \rightarrow \infty} \frac{n !}{m^{n}} h^{q}(Y, m L) ;
$$

(ii) for all $t \in \mathbb{R}_{\geq 0}$ and $M \in \operatorname{Pic}(Y)_{\mathbb{R}}$, we have $\hat{h}^{q}(Y, t M)=t^{n} \hat{h}^{q}(Y, M)$;

(iii) the function $\hat{h}^{q}(Y, \cdot)$ is continuous on any finite dimensional real subspace of $\operatorname{Pic}(Y)_{\mathbb{R}}$.

For any presentation of $M \in \operatorname{Pic}(Y)_{\mathbb{R}}$ as $M=\sum_{i} x_{i} L_{i}$ with $L_{i} \in \operatorname{Pic}(Y)$ and $x_{i} \in \mathbb{R}$, we further have

$$
\hat{h}^{q}(Y, M)=\limsup _{m \rightarrow+\infty} \frac{n !}{m^{n}} h^{q}\left(Y, \sum_{i}\left\lfloor m x_{i}\right\rfloor L_{i}\right) .
$$

If $M$ is nef, then this limsup is a limit, and $\hat{h}^{q}(Y, M)=0$ for $q>0$. 
As in the Appendix, we slightly abusively denote by $L \in \operatorname{Pic}(Y)_{\mathbb{R}}$ the image of $L \in \operatorname{Pic}(Y)$.

Proof. Set $P:=\operatorname{Pic}(Y)$. By $\S 3.3$, the function $h: P \rightarrow \mathbb{R}_{\geq 0}$ defined by $h(L):=n ! h^{q}(Y, L)$ satisfies the assumptions of Theorem A.1 with $s=n$. The existence and uniqueness of $\hat{h}^{q}$ are thus direct consequences of Theorem A.1, while (3.13) follows from Proposition A.5.

To prove the final point, we may enlarge the set $L_{1}, \ldots, L_{r} \in \operatorname{Pic}(Y)$ and assume that $L_{1}$ is ample. Then

$$
\sigma:=\left\{x \in \mathbb{R}^{r} \mid M(x):=\sum_{i} x_{i} L_{i} \text { ample }\right\}
$$

is a non-empty open convex cone, and $M(x)$ is nef if and only if $x \in \bar{\sigma}$ (since $M(x)$ nef implies that $\left(x_{1}+\varepsilon\right) L_{1}+\sum_{i>1} x_{i} L_{i}$ is ample for all $\left.\varepsilon>0\right)$. For any $x \in \mathbb{Z}^{r} \cap \sigma$, Lemma 3.10 implies $h^{q}(Y, m M(x))=0$ for $m \gg 1$ if $q>0$, while Corollary 3.11 and Lemma 3.19 yield

$$
\hat{h}^{0}(Y, M(x))=\lim _{m \rightarrow \infty} \frac{n !}{m^{n}} h^{0}(Y, m M(x)) .
$$

The final assertion of the theorem is now a consequence of Proposition A.5.

In the special case $q=0$, we define the volume of $M \in \operatorname{Pic}(Y)_{\mathbb{R}}$ as

$$
\operatorname{vol}(M):=\operatorname{vol}(Y, M):=\hat{h}^{0}(Y, M) .
$$

Lemma 3.21. Let $M \in \operatorname{Pic}(Y)_{\mathbb{R}}$ and let $E$ be a line bundle associated to an effective Cartier divisor. Then

$$
\operatorname{vol}(M) \leq \operatorname{vol}(M+E) .
$$

Proof. By continuity and homogeneity of the volume on $\operatorname{Pic}(Y)_{\mathbb{R}}$ shown in Theorem 3.20(ii) and (iii), we may assume that $M=L$ is a line bundle on $Y$. By assumption on $E$, there is a regular global section $s \in H^{0}(Y, E)$. For any $m \in \mathbb{N}$, multiplication by $s^{m}$ yields an injection of sheaves

$$
\mathcal{O}_{Y}(m L) \hookrightarrow \mathcal{O}_{Y}(m(L+E)),
$$

inducing an injection of $K^{\circ}$-modules

$$
H^{0}(Y, m L) \hookrightarrow H^{0}(Y, m(L+E)) .
$$

Thus

which implies the claim.

$$
\mathrm{c}\left(H^{0}(Y, m L)\right) \leq \mathrm{c}\left(H^{0}(Y, m(L+E))\right),
$$

Remark 3.22. We were not able to prove that (3.14) holds in case of $M \in \operatorname{Pic}(Y)_{\mathbb{R}}$ and an ample line bundle $E$. It would be quite plausible that this holds and in the Noetherian case it is true. In general, the problem is to construct a regular global section of $m E$ for some non-zero $m \in \mathbb{N}$. Then homogeneity of the volume (see Theorem 3.20(ii)) would give (3.14). In Lemma 4.1, we will solve the problem in a special case which will be enough for our application.

\section{Volume formulas for nef models and semipositive metrics}

Let $X$ be a projective scheme of dimension $n$ over a non-Archimedean field $K$. We denote the valuation of $K$ by $v$. In the first subsection, we will first prove an asymptotic volume formula for an effective vertical Cartier divisor $D$ on a model of $X$ in terms of an integral of the model function $\phi_{D}$ against a Monge-Ampère measure.

In $\left[\mathrm{BGJ}^{+} 20\right]$ and $[\mathrm{BE} 21]$, it was shown that the non-Archimedean volume $\operatorname{vol}\left(L, \phi, \phi^{\prime}\right)$ agrees with the energy for semipositive metrics $\phi, \phi^{\prime}$ of the line bundle $L$ under certain assumptions. In fact, the additional assumptions in $\left[\mathrm{BGJ}^{+} 20\right]$ were that the underlying scheme $X$ is a normal variety and that the 
non-Archimedean base field $K$ is discretely valued while in [BE21], the result holds for any non-Archimedean field, but the line bundle $L$ was assumed to be ample and $X$ was assumed to be smooth. The goal of the second subsection is to generalize both results allowing any non-Archimedean field and not requiring $L$ to be ample. Our proof uses the asymptotic volume formula and our previous results on torsion schemes.

\subsection{An asymptotic volume formula}

Let $K$ be any non-Archimedean field. In this subsection, we generalize a crucial volume formula on an effective vertical Cartier divisor $D$ of a given projective $K^{\circ}$-model $\mathscr{X}$ from the ample to the nef case. Such a formula was obtained in [BGM20] by using a change of metric formula in terms of the Deligne pairing from [BE21]. We extend the volume formula here to nef line bundles by using a continuity argument for the volumes on $D$ introduced in Section 3 as the effective vertical Cartier divisor $D$ is obviously a finitely presented projective torsion scheme over $K^{\circ}$.

In this special situation, we can really prove the desired volume inequality mentioned in Remark 3.22:

Lemma 4.1. Let $\mathscr{X}$ be a flat projective and finitely presented scheme over $K^{\circ}$. Let $L, E \in \operatorname{Pic}(\mathscr{X})_{\mathbb{R}}$ with E nef. Then for any effective vertical Cartier divisor $D$ on $\mathscr{X}$, we have

$$
\operatorname{vol}(D, L) \leq \operatorname{vol}(D, L+E) .
$$

Proof. By continuity and homogeneity of the volume ( $c f$. Theorem 3.20$)$, we may assume that $E \in \operatorname{Pic}(\mathscr{X})$ is ample and that $L \in \operatorname{Pic}(\mathscr{X})$. From Proposition 3.18, we deduce that for $m \gg 0$ the line bundle $m E$ has a relatively regular section $s$. Hence, replacing $E$ by $m E$ and $L$ by $m L$ and using homogeneity of the volume again, we can assume that $E$ has a relatively regular section $s$. It follows from [BGM20, Lemma 1.3] that $\left.s\right|_{D}$ is a regular section of $\left.E\right|_{D}$. We recall that a regular section has an associated effective Cartier divisor $\operatorname{div}\left(\left.s\right|_{D}\right)$ and $\left.E\right|_{D}$ is isomorphic to $\mathcal{O}\left(\operatorname{div}\left(\left.s\right|_{D}\right)\right)$. Then the claim follows from Lemma 3.21.

In the following, we consider a flat projective $K^{\circ}$-model $\mathscr{X}$ of the $n$-dimensional projective scheme $X$ over $K$. Let $D$ be an effective vertical Cartier divisor on $\mathscr{X}$. For any continuous function $f: X^{\text {an }} \rightarrow \mathbb{R}$, the integral

$$
\int_{X^{\mathrm{an}}} f d d^{c} \phi \mathscr{L}_{1} \wedge \cdots \wedge d d^{c} \phi \mathscr{L}_{n}
$$

is multilinear in $\mathscr{L}_{1}, \ldots, \mathscr{L}_{n} \in \operatorname{Pic}(\mathscr{X})$ and hence extends canonically to a multilinear function on $\operatorname{Pic}(\mathscr{X})_{\mathbb{R}}$.

Proposition 4.2. Let $\mathscr{L} \in \operatorname{Pic}(\mathscr{X})_{\mathbb{R}}$ be nef and let $\phi_{D}$ be the model function associated to the effective vertical Cartier divisor $D$. Then we have

$$
\operatorname{vol}(D, \mathscr{L})=\int_{X^{\text {an }}} \phi_{D}\left(d d^{c} \phi \mathscr{L}\right)^{n}
$$

Proof. If $\mathscr{L} \in \operatorname{Pic}(\mathscr{X})$ is ample, then this is proven in [BGM20, Theorem 2.4]. By homogeneity, we conclude that the claim holds for ample line bundles in $\operatorname{Pic}(\mathscr{X})_{\mathbb{Q}}$. In general, Remark 2.3 yields a finite dimensional subspace $W$ of $\operatorname{Pic}(\mathscr{X})_{\mathbb{Q}}$ such that the nef $\mathscr{L}$ is a limit of ample line bundles in $W$ and hence the claim follows from the previous case by continuity.

Proposition 4.3. Let $\mathscr{X}$ be a flat projective $K^{\circ}$-model of the $n$-dimensional projective scheme $X$ over K. Let $D$ be an effective vertical Cartier divisor on $\mathscr{X}$, let $\mathscr{L}_{0}, \ldots, \mathscr{L}_{r}$ be in $\operatorname{Pic}(\mathscr{X})$ and assume that $\mathscr{L}_{1}, \ldots, \mathscr{L}_{r}$ are nef. Then we have

$$
h^{0}\left(D, \mathscr{L}_{0}+m_{1} \mathscr{L}_{1}+\cdots+m_{r} \mathscr{L}_{r}\right)=\frac{1}{n !} \int_{X^{\text {an }}} \phi_{D}\left(d d^{c} \phi_{m_{1}} \mathscr{L}_{1}+\cdots+m_{r} \mathscr{L}_{r}\right)^{n}+o\left(m^{n}\right)
$$

for $m_{1}, \ldots, m_{r} \in \mathbb{N}$ and $m:=m_{1}+\cdots+m_{r} \rightarrow \infty$. If all $\mathscr{L}_{1}, \ldots, \mathscr{L}_{r}$ are ample, then the above asymptotic formula holds even with o $\left(m^{n}\right)$ replaced by $O\left(m^{n-1}\right)$. 
Proof. By Lemma 3.19, it is enough to consider the case $\mathscr{L}_{0}=\mathcal{O}_{\mathscr{X}}$. Then we deal first with the case where $\mathscr{L}_{1}, \ldots, \mathscr{L}_{r}$ are nef. We argue by contradiction. If the claim is not true, then there is $\varepsilon>0$ such that for any $i \in\{1, \ldots, r\}$ there is a sequence $\left(m_{i}^{(k)}\right)_{k \in \mathbb{N}}$ in $\mathbb{N}$ with $m^{(k)}=m_{1}^{(k)}+\cdots+m_{r}^{(k)} \rightarrow \infty$ and

$$
\left|h^{0}\left(D, m_{1}^{(k)} \mathscr{L}_{1}+\cdots+m_{r}^{(k)} \mathscr{L}_{r}\right)-\frac{1}{n !} \int_{X^{\text {an }}} \phi_{D}\left(d d^{c} \phi_{m_{1}^{(k)} \mathscr{L}_{1}+\cdots+m_{r}^{(k)} \mathscr{L}_{r}}\right)^{n}\right| \geq \varepsilon \cdot\left(m^{(k)}\right)^{n}
$$

for all $k \in \mathbb{N}$. Passing to a subsequence, we may assume that $m_{i}^{(k)} / m^{(k)}$ converges to a non-negative $x_{i} \in \mathbb{R}$. We deduce from Theorem 3.20 that

$$
\operatorname{vol}\left(D, x_{1} \mathscr{L}_{1}+\cdots+x_{r} \mathscr{L}_{r}\right)=\lim _{m \rightarrow \infty} \frac{h^{0}\left(D, \sum_{i=1}^{r}\left\lfloor m x_{i}\right\rfloor \mathscr{L}_{i}\right)}{m^{n} / n !} .
$$

We pick any $\delta>0$. Then we have $\left|m_{i}^{(k)}-x_{i} m^{(k)}\right| \leq \delta m^{(k)}$ for $k \gg 0$ and Lemma A.4 yields

$$
h^{0}\left(D, m_{1}^{(k)} \mathscr{L}_{1}+\cdots+m_{r}^{(k)} \mathscr{L}_{r}\right)-h^{0}\left(D,\left\lfloor m^{(k)} x_{1}\right\rfloor \mathscr{L}_{1}+\cdots+\left\lfloor m^{(k)} x_{r}\right\rfloor \mathscr{L}_{r}\right)=\delta \cdot O\left(\left(m^{(k)}\right)^{n}\right)
$$

and hence (4.2) leads to

$$
\left(m^{(k)}\right)^{-n} h^{0}\left(D, m_{1}^{(k)} \mathscr{L}_{1}+\cdots+m_{r}^{(k)} \mathscr{L}_{r}\right)-\frac{1}{n !} \operatorname{vol}\left(D, x_{1} \mathscr{L}_{1}+\cdots+x_{r} \mathscr{L}_{r}\right)=\mathcal{O}(\delta)
$$

for $k$ sufficiently large. By Proposition 4.2 , we may replace vol $\left(D, x_{1} \mathscr{L}_{1}+\cdots+x_{r} \mathscr{L}_{r}\right)$ by

$$
\int_{X^{a n}} \phi_{D}\left(d d^{c} \phi_{x_{1} \mathscr{L}_{1}+\cdots+x_{r} \mathscr{L}_{r}}\right)^{n}
$$

If we choose $\delta$ sufficiently small, we get a contradiction to (4.1).

If $\mathscr{L}_{1}, \ldots, \mathscr{L}_{r}$ are ample, then Corollary 3.11 and Lemma 3.19 show that $h^{0}\left(D, \mathscr{L}_{0}+m_{1} \mathscr{L}_{1}+\cdots+m_{r} \mathscr{L}_{r}\right)$ is a polynomial function of degree at most $n$ in $m_{1}, \ldots, m_{r}$ for $m \gg 0$. Since

$$
\int_{X^{\text {an }}} \phi_{D}\left(d d^{c} \phi_{m_{1}^{(k)} \mathscr{L}_{1}+\cdots+m_{r}^{(k)} \mathscr{L}_{r}}\right)^{n}
$$

is also a polynomial function of degree at most $n$, the difference of the two functions is not only of order $o\left(m^{n}\right)$, but even of order $O\left(m^{n-1}\right)$.

\subsection{Comparison between energy and non-Archimedean volume}

The following easy filtration argument will be applied several times.

Lemma 4.4. Let $\mathscr{X}$ be a flat proper scheme over $K^{\circ}$ with a line bundle $\mathscr{M}$ and an effective vertical Cartier divisor E. Then we have

$$
\mathrm{c}\left(\frac{H^{0}(\mathscr{X}, \mathscr{M}+m \mathcal{O}(E))}{H^{0}(\mathscr{X}, \mathscr{M})}\right) \leq \sum_{i=1}^{m} \mathrm{c}\left(H^{0}(E, \mathscr{M}+i \mathcal{O}(E))\right)
$$

for any $m \in \mathbb{N}$.

Let $X$ be the generic fiber of $\mathscr{X}$ and let $M=\left.\mathscr{M}\right|_{X}$. Recall from Remark 2.13 that on the left hand side we have a quotient of two lattices in $H^{0}(X, M)$ with content $\ell$ defined by 2.9 .

Proof. We may assume $m>0$. Then for every $i \in\{1, \ldots m\}$, multiplication with the canonical global section $s_{E}$ leads to a short exact sequence

$$
\left.0 \longrightarrow \mathcal{O}_{\mathscr{X}} \stackrel{\cdot_{E}}{\longrightarrow} \mathcal{O}(E) \longrightarrow \mathcal{O}(E)\right|_{E} \rightarrow 0
$$

inducing a short exact sequence

$$
0 \longrightarrow \mathscr{M}+(i-1) \mathcal{O}(E) \longrightarrow \mathscr{M}+\left.i \mathcal{O}(E) \longrightarrow(\mathscr{M}+i \mathcal{O}(E))\right|_{E} \rightarrow 0
$$


The start of the corresponding long exact cohomology sequence is

$$
0 \longrightarrow H^{0}(\mathscr{X}, \mathscr{M}+(i-1) \mathcal{O}(E)) \longrightarrow H^{0}(\mathscr{X}, \mathscr{M}+i \mathcal{O}(E)) \longrightarrow H^{0}(E, \mathscr{M}+i \mathcal{O}(E)) \longrightarrow \ldots
$$

and hence we get

$$
\mathrm{c}\left(\frac{H^{0}(\mathscr{X}, \mathscr{M}+i \mathcal{O}(E))}{H^{0}(\mathscr{X}, \mathscr{M}+(i-1) \mathcal{O}(E))}\right) \leq \mathrm{c}\left(H^{0}(E, \mathscr{M}+i \mathcal{O}(E))\right)
$$

leading to

$$
\mathrm{c}\left(\frac{H^{0}(\mathscr{X}, \mathscr{M}+m \mathcal{O}(E))}{H^{0}(\mathscr{X}, \mathscr{M})}\right) \leq \sum_{i=1}^{m} \mathrm{c}\left(H^{0}(E, \mathscr{M}+i \mathcal{O}(E))\right)
$$

and proving the claim.

In the following result, we will use the content $\mathrm{c}\left(\mathcal{V}_{1} / \mathcal{V}_{2}\right)$ of the virtual quotient of two lattices $\mathcal{V}_{1}, \mathcal{V}_{2}$ in the same $K$-vector space. We refer to 2.9 for the definition.

We first deal with the model case.

Proposition 4.5. Let $L$ be a line bundle on the projective scheme $X$ and let $\mathscr{X}$ be a projective model of X. We consider nef models $\mathscr{L}_{1}$ and $\mathscr{L}_{2}$ of $L$ on $\mathscr{X}$ and we write $\mathscr{L}_{1}-\mathscr{L}_{2}=\mathcal{O}(D)$ for some vertical Cartier divisor $D$ on $\mathscr{X}$. In addition, let $\mathscr{M}$ be a line bundle on $\mathscr{X}$ with generic fibre $M:=\mathscr{M}_{\mid X}$. Then we have

$$
E\left(L, \phi_{\mathscr{L}_{1}}, \phi_{\mathscr{L}_{2}}\right)=\lim _{m \rightarrow 0} \frac{n !}{m^{n+1}} \mathrm{c}\left(\frac{H^{0}\left(\mathscr{X}, \mathscr{M}+m \mathscr{L}_{1}\right)}{H^{0}\left(\mathscr{X}, \mathscr{M}+m \mathscr{L}_{2}\right)}\right) .
$$

Proof. We first study what happens if we replace $D$ by $D^{\prime}:=D+\operatorname{div}(\pi)$ for any non-zero $\pi \in K$. Then we replace the model $\mathscr{L}_{1}$ by the model $\mathscr{L}_{1}^{\prime}:=\mathscr{L}_{1}(\operatorname{div}(\pi)) \simeq \mathscr{L}_{1}$ of $L$ which is also nef. By construction, we have $\mathcal{O}\left(D^{\prime}\right)=\mathscr{L}_{1}^{\prime}-\mathscr{L}_{2}$. Note $\phi_{\mathscr{L}_{1}^{\prime}}=v(\pi)+\phi \mathscr{L}_{1}$. Using Proposition 2.29(e), we get

$$
E\left(L, \phi_{\mathscr{L}_{1}^{\prime}}, \phi_{\mathscr{L}_{2}}\right)=v(\pi) \operatorname{deg}_{L}(X)+E\left(L, \phi_{\mathscr{L}_{1}}, \phi_{\mathscr{L}_{2}}\right) .
$$

We have

$$
\mathrm{c}\left(\frac{H^{0}\left(\mathscr{X}, \mathscr{M}+m \mathscr{L}_{1}^{\prime}\right)}{H^{0}\left(\mathscr{X}, \mathscr{M}+m \mathscr{L}_{1}\right)}\right)=v(\pi) m h^{0}(X, M+m L)
$$

and hence

$$
\mathrm{c}\left(\frac{H^{0}\left(\mathscr{X}, \mathscr{M}+m \mathscr{L}_{1}^{\prime}\right)}{H^{0}\left(\mathscr{X}, \mathscr{M}+m \mathscr{L}_{2}\right)}\right)=c\left(\frac{H^{0}\left(\mathscr{X}, \mathscr{M}+m \mathscr{L}_{1}\right)}{H^{0}\left(\mathscr{X}, \mathscr{M}+m \mathscr{L}_{2}\right)}\right)+C_{n}(m)
$$

with $C_{n}(m)$ defined as

$$
v(\pi) m h^{0}\left(X, M \otimes L^{\otimes m}\right)=v(\pi) m h^{0}\left(X, L^{\otimes m}\right)+o\left(m^{n+1}\right)=v(\pi) \operatorname{vol}(L) \frac{m^{n+1}}{n !}+o\left(m^{n+1}\right)
$$

where we used the analogue of (3.6) in Lemma 3.19 for projective schemes over a field (see $\left[\mathrm{BGJ}^{+} 20\right.$, Proposition 3.1.2]). Since $L$ is a nef line bundle on $X$, we know that $\operatorname{vol}(L)=\operatorname{deg}_{L}(X)$ and hence the claim for $D^{\prime}$ implies the claim for $D$. This proves that we can replace $D$ by $D^{\prime}$.

First, we will prove the inequality

$$
\limsup _{m \rightarrow 0} \frac{n !}{m^{n+1}} c\left(\frac{H^{0}\left(\mathscr{X}, \mathscr{M}+m \mathscr{L}_{1}\right)}{H^{0}\left(\mathscr{X}, \mathscr{M}+m \mathscr{L}_{2}\right)}\right) \leq E\left(L, \phi \mathscr{L}_{1}, \phi \mathscr{L}_{2}\right)
$$

There is a non-zero $\pi$ in $K^{\circ}$ such that $D+\operatorname{div}(\pi)$ is an effective Cartier divisor. Replacing $D$ by $D+\operatorname{div}(\pi)$, the above shows that it is enough to prove (4.3) if $D$ is effective.

For non-zero $m$, Lemma 4.4 applied with $\mathscr{M}+m \mathscr{L}_{2}$ instead of $\mathscr{M}$ and with $D$ instead of $E$ shows that

$$
\mathrm{c}\left(\frac{H^{0}\left(\mathscr{X}, \mathscr{M}+m \mathscr{L}_{1}\right)}{H^{0}\left(\mathscr{X}, \mathscr{M}+m \mathscr{L}_{2}\right)}\right) \leq \sum_{i=1}^{m} \mathrm{c}\left(H^{0}\left(D, \mathscr{M}+m \mathscr{L}_{2}+i \mathcal{O}(D)\right)\right) .
$$


Using $\mathscr{L}_{1}-\mathscr{L}_{2}=\mathcal{O}(D)$, we get

$$
\mathrm{c}\left(\frac{H^{0}\left(\mathscr{X}, \mathscr{M}+m \mathscr{L}_{1}\right)}{H^{0}\left(\mathscr{X}, \mathscr{M}+m \mathscr{L}_{2}\right)}\right) \leq \sum_{i=1}^{m} \mathrm{c}\left(H^{0}\left(D, \mathscr{M}+i \mathscr{L}_{1}+(m-i) \mathscr{L}_{2}\right)\right) .
$$

Since $\mathscr{L}_{1}$ and $\mathscr{L}_{2}$ are nef, Proposition 4.3 shows that the right hand side is

$$
\sum_{i=1}^{m} \sum_{j_{1}+j_{2}=n} \frac{i^{j_{1}}(m-i)^{j_{2}}}{j_{1} ! j_{2} !} \int_{X^{\text {an }}} \phi_{D}\left(d d^{c} \phi \mathscr{L}_{1}\right)^{j_{1}} \wedge\left(d d^{c} \phi \mathscr{L}_{2}\right)^{j_{2}}+o\left(m^{n+1}\right) .
$$

We note that the following limit for $m \rightarrow \infty$ exists and is given by the sum of Riemann integrals

$$
\lim _{m \rightarrow \infty} m^{-(n+1)} \sum_{i=1}^{m} i^{j_{1}}(m-i)^{j_{2}}=\lim _{m \rightarrow \infty} \frac{1}{m} \sum_{i=1}^{m}\left(\frac{i}{m}\right)^{j_{1}}\left(1-\frac{i}{m}\right)^{j_{2}}=\int_{0}^{1} t^{j_{1}}(1-t)^{j_{2}} d t .
$$

Using the identity $\int_{0}^{1} t^{j_{1}}(1-t)^{j_{2}} d t=\frac{j_{1} ! j_{2} !}{(n+1) !}$, we get

$$
\sum_{i=1}^{m} \frac{{ }_{i}^{j_{1}}(m-i)^{j_{2}}}{j_{1} ! j_{2} !}=\frac{m^{n+1}}{(n+1) !}+o\left(m^{n+1}\right)
$$

Using our previous considerations, we get

$$
\begin{aligned}
\limsup _{m \rightarrow 0} \frac{n !}{m^{n+1}} c\left(\frac{H^{0}\left(\mathscr{X}, \mathscr{M}+m \mathscr{L}_{1}\right)}{H^{0}\left(\mathscr{X}, \mathscr{M}+m \mathscr{L}_{2}\right)}\right) \\
\leq \frac{1}{n+1} \sum_{j_{1}+j_{2}=n} \int_{X^{\text {an }}} \phi_{D}\left(d d^{c} \phi \mathscr{L}_{1}\right)^{j_{1}} \wedge\left(d d^{c} \phi \mathscr{L}_{2}\right)^{j_{2}} .
\end{aligned}
$$

By definition, the right hand side is $E\left(L, \phi_{\mathscr{L}_{1}}, \phi_{\mathscr{L}_{2}}\right)$. This proves (4.3).

If we multiply (4.3) by -1 and if we exchange $\mathscr{L}_{1}$ with $\mathscr{L}_{2}$, then we get the reverse inequality

$$
\liminf _{m \rightarrow 0} \frac{n !}{m^{n+1}} c\left(\frac{H^{0}\left(\mathscr{X}, \mathscr{M}+m \mathscr{L}_{1}\right)}{H^{0}\left(\mathscr{X}, \mathscr{M}+m \mathscr{L}_{2}\right)}\right) \geq E\left(L, \phi \mathscr{L}_{1}, \phi \mathscr{L}_{2}\right)
$$

Combining (4.3) and (4.4), we get the claim in the proposition.

Theorem 4.6. Let $L$ be a line bundle on a geometrically reduced proper scheme $X$ over $K$ and let $\phi_{1}$ and $\phi_{2}$ be continuous semipositive metrics on $L^{\text {an }}$. Then we have

$$
\operatorname{vol}\left(L, \phi_{1}, \phi_{2}\right)=E\left(L, \phi_{1}, \phi_{2}\right) \text {. }
$$

Proof. By base change and using Proposition 2.37 and Proposition 2.29, we may assume that $K$ is algebraically closed. By Chow's lemma and birational invariance of non-Archimedean volumes and energy (see Lemma 2.36 and Proposition 2.29), we may assume $X$ to be projective.

We first prove the claim for semipositive model metrics. Since the projective models of $X$ which are integrally closed in $X$ are cofinal by Lemma 2.21 , we may assume that $\phi_{i}=\phi_{\mathscr{L}_{i}}$ for some nef $\mathbb{Q}$-line bundle $\mathscr{L}_{i}$ on some common projective model $\mathscr{X}$ which is integrally closed in $X$. Homogeneity of the non-Archimedean volume in Corollary 2.39 and the energy in Proposition 2.29 show that we may assume that the $\mathscr{L}_{i}$ are nef line bundles on $\mathscr{X}$. Then the claim follows from Proposition 4.5, Lemma 2.19 and Remark 2.35.

Arbitrary continuous semipositive metrics on $L^{\text {an }}$ are uniform limits of semipositive model metrics on $L^{\text {an }}$. Then the claim follows from the first case as both the non-Archimedean volume and the energy are continuous in $\left(\phi_{1}, \phi_{2}\right)$ (see Propositions 2.29 and 2.33). 


\section{Differentiability of non-Archimedean volumes}

We will prove our main result about differentiation of non-Archimedean volumes. It generalizes $\left[\mathrm{BGJ}{ }^{+} 20\right.$, Theorem B] from the case of discrete valuations to arbitrary non-Archimedean complete absolute values and [BGM20, Theorem A] from the ample to the nef case.

\subsection{Intermediate result for models}

We consider an $n$-dimensional projective scheme $X$ over $K$ with a projective model $\mathscr{X}$ over $K^{\circ}$. Let $D$ be a vertical Cartier divisor on $\mathscr{X}$. Since $\mathscr{X}$ is projective, we can write $\mathcal{O}(D)=\mathscr{M}_{1}-\mathscr{M}_{2}$ for nef line bundles $\mathscr{M}_{1}, \mathscr{M}_{2}$ on $\mathscr{X}$. We consider a nef line bundle $\mathscr{L}$ and an arbitrary line bundle $\mathcal{N}$ on $\mathscr{X}$.

Lemma 5.1. Unter the above assumptions, let $\phi_{D}$ be the model function associated to the vertical Cartier divisor $D$ and let $\mathcal{F}_{j, m}:=\mathcal{N}+m \mathscr{L}+j\left(\mathscr{M}_{1}-\mathscr{M}_{2}\right)$. If $D$ is effective, then we have

$$
\frac{n !}{m^{n}} \mathrm{c}\left(\frac{H^{0}\left(\mathscr{X}, \mathcal{F}_{j+1, m}\right)}{H^{0}\left(\mathscr{X}, \mathcal{F}_{j, m}\right)}\right) \leq \int_{X^{\text {an }}} \phi_{D}\left(d d^{c} \phi_{\mathscr{L}}+d d^{c} \phi_{\mathscr{M}_{1}}\right)^{n}+o(1)
$$

for integers $m \rightarrow \infty$ and all $j \in\{0, \ldots, m-1\}$. If $-D$ is effective, then $\geq$ holds in (5.1).

Proof. We prove first the claim in the case $E:=-D$ effective. The canonical section $s_{E}$ of $\mathcal{O}(E)=\mathscr{M}_{2}-\mathscr{M}_{1}$ determines a short exact sequence of coherent sheaves on $\mathscr{X}$ :

$$
\left.0 \longrightarrow \mathcal{F}_{j+1, m} \stackrel{\otimes s_{E}}{\longrightarrow} \mathcal{F}_{j, m} \longrightarrow \mathcal{F}_{j, m}\right|_{E} \longrightarrow 0
$$

The start of the associated long exact sequence in cohomology is

$$
0 \longrightarrow H^{0}\left(\mathscr{X}, \mathcal{F}_{j+1, m}\right) \stackrel{\otimes S_{E}}{\longrightarrow} H^{0}\left(\mathscr{X}, \mathcal{F}_{j, m}\right) \longrightarrow H^{0}\left(E, \mathcal{F}_{j, m}\right) \longrightarrow \cdots
$$

and hence

$$
\mathrm{c}\left(H^{0}\left(\mathscr{X}, \mathcal{F}_{j, m}\right) / H^{0}\left(\mathscr{X}, \mathcal{F}_{j+1, m}\right)\right) \leq \mathrm{c}\left(H^{0}\left(E, \mathcal{F}_{j, m}\right)\right)=h^{0}\left(E, \mathcal{F}_{j, m}\right) .
$$

Using that $\mathscr{M}_{1}$ and $\mathscr{M}_{2}$ are nef, we deduce from Lemma 4.1 that

$$
\operatorname{vol}\left(E, \mathcal{F}_{j, m}\right) \leq \operatorname{vol}\left(E, \mathcal{N}+m \mathscr{L}+j \mathscr{M}_{1}\right) \leq \operatorname{vol}\left(E, \mathcal{N}+m\left(\mathscr{L}+\mathscr{M}_{1}\right)\right) .
$$

These inequalities and Proposition 4.3 give

$$
h^{0}\left(E, \mathcal{F}_{j, m}\right) \leq \frac{m^{n}}{n !} \int_{X^{\mathrm{an}}} \phi_{E}\left(d d^{c} \phi \mathscr{L}+d d^{c} \phi_{\mathscr{M}_{1}}\right)^{n}+o\left(m^{n}\right)
$$

By (5.2), we get

$$
\frac{n !}{m^{n}} \mathrm{c}\left(H^{0}\left(\mathscr{X}, \mathcal{F}_{j, m}\right) / H^{0}\left(\mathscr{X}, \mathcal{F}_{j+1, m}\right)\right) \leq \int_{X^{\text {an }}} \phi_{E}\left(d d^{c} \phi \mathscr{L}+d d^{c} \phi_{\mathscr{M}_{1}}\right)^{n}+o(1) .
$$

Using that $\phi_{D}=-\phi_{E}$, we get the desired reverse inequality in (5.1).

Now we deal with the case $E:=D$ effective. The proof is quite similar as in the first case. The canonical global section $s_{E}$ induces a short exact sequence

$$
\left.0 \longrightarrow \mathcal{F}_{j, m} \stackrel{\otimes s_{E}}{\longrightarrow} \mathcal{F}_{j+1, m} \longrightarrow \mathcal{F}_{j+1, m}\right|_{E} \longrightarrow 0
$$

of coherent sheaves on $\mathscr{X}$. The same argument with the long exact cohomology sequence gives

$$
\mathrm{c}\left(H^{0}\left(\mathscr{X}, \mathcal{F}_{j+1, m}\right) / H^{0}\left(\mathscr{X}, \mathcal{F}_{j, m}\right)\right) \leq h^{0}\left(E, \mathcal{F}_{j+1, m}\right) .
$$

The asymptotic formula (5.3) holds still for $j+1$ instead of $j$ and so we get (5.1) by using (5.4). 


\subsection{Main result}

In this subsection, we assume that $X$ is an $n$-dimensional geometrically reduced proper scheme over $K$. We apply first the previous result to model functions.

Lemma 5.2. Let $L$ be a line bundle on $X, f$ a model function on $X^{\text {an }}$ and $\phi$ a continuous semipositive metric on L. Let $M$ be a line bundle on $X$ and $\phi_{1}, \phi_{2}$ continuous semipositive metrics of $M$ such that $f=\phi_{1}-\phi_{2}$. If $f \geq 0$, then

$$
\operatorname{vol}(L, \phi+f, \phi) \leq \int_{X^{\text {an }}} f\left(d d^{c} \phi+d d^{c} \phi_{1}\right)^{n}
$$

If $f \leq 0$, then $\geq$ holds in (5.5).

Proof. By Corollary 2.37, the left hand side is invariant under base change. By Proposition 2.23(f), the right hand side is invariant under base change. Hence we can assume that $K$ is algebraically closed. By Chow's lemma and birational invariance of non-Archimedean volumes and energy (see Lemma 2.36 and Proposition 2.29), we may assume $X$ projective. By continuity of the non-Archimedean volume and of the non-Archimedean Monge-Ampère measures, we may assume that $\phi, \phi_{1}, \phi_{2}$ are induced by nef $\mathbb{Q}$-line bundles $\mathscr{L}, \mathscr{M}_{1}, \mathscr{M}_{2}$. Using Lemma 2.21 , we may assume that the $\mathbb{Q}$-line bundles are determined on a common projective model $\mathscr{X}$ which is integrally closed in $X$. By the homogenity of the non-Archimedean volume, we may assume that $\mathscr{L}, \mathscr{M}_{1}, \mathscr{M}_{2}$ are honest line bundles on $\mathscr{X}$.

Let us assume first that $f \geq 0$. By our above assumptions, we have a vertical Cartier divisor $D$ on $\mathscr{X}$ with $\phi_{D}=f$. Since $\mathscr{X}$ is integrally closed, Lemma 2.20 shows that $D$ is an effective Cartier divisor. We have to prove that

$$
\limsup _{m \rightarrow \infty} \frac{1}{m^{n+1} / n !} \operatorname{vol}\left(\|\cdot\|_{m\left(\phi_{\mathscr{L}}+\phi_{D}\right)},\|\cdot\|_{m \phi_{\mathscr{L}}}\right) \leq \int_{X^{\text {an }}} \phi_{D}\left(d d^{c} \phi_{\mathscr{L}}+d d^{c} \phi_{\mathscr{M}_{1}}\right)^{n} .
$$

By Remark 2.35 and Lemma 2.19, it is enough to prove that

$$
\limsup _{m \rightarrow \infty} \frac{1}{m^{n+1} / n !} \mathrm{c}\left(\frac{H^{0}\left(\mathscr{X}, m\left(\mathscr{L}+\mathscr{M}_{1}-\mathscr{M}_{2}\right)\right)}{H^{0}(\mathscr{X}, m \mathscr{L})}\right) \leq \int_{X^{\text {an }}} \phi_{D}\left(d d^{c} \phi \mathscr{L}+d d^{c} \phi_{\mathscr{M}_{1}}\right)^{n} .
$$

We apply now Lemma 5.1 with $\mathcal{N}=\mathcal{O}_{\mathscr{L}}, \mathscr{L}$ and $\mathcal{O}(D)=\mathscr{M}_{1}-\mathscr{M}_{2}$ for the summands in

$$
\mathrm{c}\left(\frac{H^{0}\left(\mathscr{X}, m\left(\mathscr{L}+\mathscr{M}_{1}-\mathscr{M}_{2}\right)\right)}{H^{0}(\mathscr{X}, m \mathscr{L})}\right)=\sum_{j=0}^{m-1} \mathrm{c}\left(\frac{H^{0}\left(\mathscr{X}, \mathcal{F}_{j+1, m}\right)}{H^{0}\left(\mathscr{X}, \mathcal{F}_{j, m}\right)}\right) .
$$

This gives (5.6) and proves the case $f \geq 0$. Now assume that $f \leq 0$. Then the reverse inequality in (5.5) follows by applying the first case for $-f$ switching the role of $\mathscr{L}_{1}, \mathscr{L}_{2}$.

Theorem 5.3. Let $X$ be an $n$-dimensional geometrically reduced proper scheme over $K$. Let $L$ be a line bundle on $X, f$ a continuous real function on $X^{\text {an }}$ and $\phi$ a continuous semipositive metric on $L$. Then

$$
\left.\frac{d}{d t}\right|_{t=0} \operatorname{vol}(L, \phi+t f, \phi)=\int_{X^{\text {an }}} f\left(d d^{c} \phi\right)^{n} .
$$

Proof. We have seen in 2.15 that model functions are dense in the space of continuous functions on $X^{\text {an }}$. By continuity and monoticity of non-Archimedean volumes in Proposition 2.33, we may assume that $f$ is a model function (see the proofs of $\left[\mathrm{BGJ}^{+} 20\right.$, Theorem 5.4.3] or [BGM20, Theorem 3.1] for details). It follows from 2.15 that $L$ is nef and hence $\operatorname{vol}(L)=\operatorname{deg}_{L}(X)$. If $f \leq 0$, then Lemma 5.2 implies

$$
\liminf _{t \rightarrow 0+} \frac{\operatorname{vol}(L, \phi+t f, \phi)}{t} \geq \int_{X^{\text {an }}} f\left(d d^{c} \phi\right)^{n} .
$$


Using that for $c \in \mathbb{R}$, we have $\operatorname{vol}(L, \phi+c, \phi)=\operatorname{vol}(L)+c \operatorname{deg}_{L}(X)$ (see Proposition 2.33), we deduce from Proposition 2.23(e) that (5.7) holds for any continuous function $f$. Using Lemma 5.2 in the case $f \geq 0$, a similar trick shows that

$$
\limsup _{t \rightarrow 0+} \frac{\operatorname{vol}(L, \phi+t f, \phi)}{t} \leq \int_{X^{\text {an }}} f\left(d d^{c} \phi\right)^{n}
$$

holds for any continuous function $f$ and hence we get

$$
\lim _{t \rightarrow 0+} \frac{\operatorname{vol}(L, \phi+f t, \phi)}{t}=\int_{X^{\text {an }}} f\left(d d^{c} \phi\right)^{n} .
$$

Finally replacing $f$ by $-f$, we get the same for $t \rightarrow 0-$ proving the claim.

\section{Orthogonality property}

In this section, we prove the orthogonality property of a continuous semipositive metric $\phi$ assuming that the semipositive envelope $\mathrm{P}(\phi)$ is continuous.

\subsection{Semipositive envelope}

Let $L$ be a line bundle on a proper scheme $X$ over $K$. We will introduce the semipositive envelope $\mathrm{P}(\psi)$ of a bounded metric $\psi$ on $L^{\text {an }}$. We always assume that $L$ has at least one semipositive model metric. This implies that $L$ is nef (see 2.15) and holds at least for semiample line bundles.

Recall from 2.10 that we use additive notation for metrics and hence $\phi \leq \psi$ for metrics on $L^{\text {an }}$ means $|\cdot|_{\phi} \geq|\cdot|_{\psi}$ for the corresponding norms on fibers.

Definition 6.1. The semipositive envelope $\mathrm{P}(\psi)$ of a bounded metric $\psi$ on $L^{\text {an }}$ is defined by

$$
\mathrm{P}(\psi):=\sup \left\{\phi \mid \phi \text { is a continuous semipositive metric on } L^{\text {an }} \text { and } \phi \leq \psi\right\} \text {. }
$$

Remark 6.2. Note that in the above definition, we may restrict our attention to semipositive model metrics of $L$ using that every semipositive continuous metric is a uniform limit of continuous semipositive metrics. It follows from the assumed existence of a semipositive model metric that $\mathrm{P}(\psi)$ is a bounded metric on $L$.

If $\mathrm{P}(\psi)$ is a continuous metric on $L^{\text {an }}$, then it follows from Dini's theorem that $\mathrm{P}(\psi)$ is a continuous semipositive metric on $L^{\text {an }}$. If $\psi$ is a continuous metric, continuity of $\mathrm{P}(\psi)$ is not clear. This property is expected in case of semiample line bundles on a normal projective variety (see [BE21, Conjecture 7.31]).

We refer to $[\mathrm{BJ} 18, \S 5.3,5.4]$ and $[\mathrm{BE} 21, \S 7.5]$ for the study of the psh-envelope which agrees with the semipositive envelope at least in the case of a continuous metric on an ample line bundle. For a continuous metric $\psi$ of $L$, the definition of $\mathrm{P}(\psi)$ agrees with $\left[\mathrm{BGJ}^{+} 20\right.$, Definition 2.5.1] where multiplicative notation for metrics was used.

Proposition 6.3. Let $\psi, \psi_{1}, \psi_{2}$ be bounded metrics on $L^{\text {an }}$. Then we have:

(a) $\mathrm{P}(\psi) \leq \psi$ with equality if $\psi$ is a continuous semipositive metric.

(b) If $a \in \mathbb{N}$, then $\mathrm{P}(a \psi)=a \mathrm{P}(\psi)$.

(c) If $\psi_{1} \leq \psi_{2}$, then $\mathrm{P}\left(\psi_{1}\right) \leq \mathrm{P}\left(\psi_{2}\right)$.

(d) If $c \in \mathbb{R}$, then $\mathrm{P}(\psi+c)=\mathrm{P}(\psi)+c$.

(e) $\sup _{X^{\text {an }}}\left|\mathrm{P}\left(\psi_{1}\right)-\mathrm{P}\left(\psi_{2}\right)\right| \leq \sup _{X^{\text {an }}}\left|\psi_{1}-\psi_{2}\right|$.

Proof. Properties (a)-(d) are obvious from the definition and (e) follows from (b)-(d).

We generalize now $\left[\mathrm{BGJ}^{+}\right.$20, Proposition 6.2.1] and [BE21, Proposition 9.11(vi)].

Proposition 6.4. Let $\psi$ be bounded metric on $L^{\text {an }}$. 
(a) We have $\|\cdot\|_{\mathrm{P}(\psi)}=\|\cdot\|_{\psi}$ for the corresponding supremum seminorms on $H^{0}(X, L)$.

(b) If $X$ is reduced, then we get $\operatorname{vol}(L, \phi, \psi)=\operatorname{vol}(L, \mathrm{P}(\phi), \mathrm{P}(\psi))$ for any bounded metric $\phi$ on $L^{\mathrm{an}}$.

Proof. By Proposition 6.3(a), we deduce $\|\cdot\|_{\mathrm{P}(\psi)} \geq\|\cdot\|_{\psi}$. We prove the converse inequality by contradiction. Assume that there is $s \in H^{0}(X, L)$ and $x \in X^{\text {an }}$ with $|s(x)|_{\mathrm{P}(\psi)}>\|s\|_{\psi}$. Rescaling the metric $\psi$ and using Proposition 6.3(d), we may assume

$$
|s(x)|_{\mathrm{P}(\psi)}>1=\|s\|_{\psi} .
$$

This yields $g:=\psi \circ s \geq 0$ and hence we get a singular metric $\psi_{s}:=\psi-g \leq \psi$ on $L^{\text {an }}$. We note that the results of [BGJ $\left.{ }^{+} 20, \S 6.1\right]$ hold over any (non-trivially valued) non-Archimedean field, as the crucial reference [GM19] works in this setting. For a continuous semipositive metric $\psi_{1} \leq \psi$ on $L^{\text {an }}$, it follows from $\left[\mathrm{BGJ}^{+} 20\right.$, Lemma 6.1.3] that $\psi^{\prime}:=\max \left(\psi_{1}, \psi_{s}\right)$ is a continuous semipositive metric on $L^{\text {an }}$ with $\psi_{1} \leq \psi^{\prime} \leq \psi$ and hence

$$
\psi^{\prime} \leq \mathrm{P}(\psi)
$$

by definition of the semipositive envelope. By construction, we have $\psi_{s} \circ s \equiv 0$ and hence (6.2) yields

$$
\mathrm{P}(\psi) \circ s \geq \psi^{\prime} \circ s=\max \left(\psi_{1} \circ s, \psi_{s} \circ s\right) \geq 0
$$

which contradicts the strict inequality in (6.1). This proves $\|\cdot\|_{\mathrm{P}(\psi)}=\|\cdot\|_{\psi}$.

Finally, (b) follows from the definition of the non-Archimedean volume and by applying (a) to the bounded metrics $m \psi$ and to $m \phi$ for any $m \in \mathbb{N}$.

The following generalizes [BGJ ${ }^{+}$20, Corollary 6.2.2] and [BE21, Corollary 9.16].

Corollary 6.5. Let L be a line bundle over a geometrically reduced proper scheme X over K. Assume that $\psi$, $\phi$ are continuous metrics on $L^{\text {an }}$ and assume that $P(\psi), P(\phi)$ are also continuous metrics on $L^{\text {an }}$. Then we have

$$
\operatorname{vol}(L, \phi, \psi)=\mathrm{E}(L, \mathrm{P}(\phi), \mathrm{P}(\psi)) .
$$

Proof. This follows by combining Proposition 6.4 and Theorem 4.6.

\subsection{Orthogonality}

In this subsection, we consider a line bundle $L$ on a proper scheme $X$ of dimension $n$ over $K$.

Definition 6.6. Let $\phi$ be a continuous metric on $L^{\text {an }}$ with $\mathrm{P}(\phi)$ a continuous metric on $L^{\text {an }}$ as well. We say the $\phi$ satisfies the orthogonality property if

$$
\int_{X^{\text {an }}}(\mathrm{P}(\phi)-\phi)\left(d d^{c} \mathrm{P}(\phi)\right)^{n}=0
$$

Theorem 6.7. We assume that $X$ is geometrically reduced. Let $\phi$ be a continuous metric on $L^{\text {an }}$ such that $\mathrm{P}(\phi)$ is also a continuous metric. Then $\phi$ satisfies the orthogonality property.

Proof. This follows from Theorem 5.3 and Proposition 6.4 by the same arguments as in the proof of $\left[\mathrm{BGJ}^{+} 20\right.$, Theorem 6.3.2].

Remark 6.8. Assume that the semipositive envelope of any continuous metric $\phi$ on $L$ is continuous. Fixing a continuous reference metric $\psi$ of $L$, orthogonality for all continuous metrics $\phi$ of $L$ is equivalent to differentiability of $\phi \mapsto E(L, \mathrm{P}(\phi), \mathrm{P}(\psi))$ for all continuous metrics $\phi$ of $L$, see [BGM20, Lemma 3.5] for the argument. This differentiability is a crucial property in the proof of the existence of solutions of non-Archimedean Monge-Ampère equations, see [BFJ15]. 


\section{Appendix A. Asymptotical functions}

In what follows, $P$ denotes an arbitrary abelian group, and we set as usual $P_{\mathbb{R}}:=P \otimes_{\mathbb{Z}} \mathbb{R}$. We use the norm $|x|:=\sum_{i}\left|x_{i}\right|$ for $x \in \mathbb{R}^{r}$.

The goal of this appendix is to establish the following elementary result.

Theorem A.1. Let $h: P \rightarrow \mathbb{R}, s \in \mathbb{R}_{>0}$, and assume that for all $L_{0}, \ldots, L_{r} \in P$ and $m=\left(m_{1}, \ldots, m_{r}\right) \in \mathbb{Z}^{r}$ we have

$$
h\left(m_{1} L_{1}+\cdots+m_{r} L_{r}\right)=O\left(|m|^{s}\right)
$$

and

$$
h\left(L_{0}+m_{1} L_{1}+\cdots+m_{r} L_{r}\right)-h\left(m_{1} L_{1}+\cdots+m_{r} L_{r}\right)=O\left(|m|^{s-1}\right) .
$$

Then there is a unique function $\hat{h}: P_{\mathbb{R}} \rightarrow \mathbb{R}$ such that:

(i) for any $L \in P$ we have

$$
\hat{h}(L)=\limsup _{m \rightarrow+\infty} m^{-s} h(m L) ;
$$

(ii) $\hat{h}$ is homogeneous of degree $s$, i.e. $\hat{h}(t M)=t^{s} \hat{h}(M)$ for $t \in \mathbb{R}_{+}$and $M \in P_{\mathbb{R}}$;

(iii) $\hat{h}$ is continuous on any finite dimensional real subspace of $P_{\mathbb{R}}$.

To simplify the notation, we slightly abusively denote by $L \in P_{\mathbb{R}}$ the image of $L \in P$. The above abstract setting is inspired by the following example.

Example A.2. Let $Y$ be a projective scheme over a field $k$ of dimension $n$. For $q \in\{0, \ldots, n\}$, the function $L \mapsto h^{q}(Y, L)$ on $\operatorname{Pic}(Y)$ satisfies (A.1) and (A.2) with $s=n$. Up to a factor $n$ !, the induced function $\hat{h}$ is then Küronya's higher cohomological function $\hat{h}^{q}: \operatorname{Pic}(Y)_{\mathbb{R}} \rightarrow \mathbb{R}_{\geq 0}$, which coincides with the volume of line bundles for $q=0$. We refer to $\left[\mathrm{BGJ}^{+} 20, \S 3.4\right]$ for details.

In this paper, we will apply the appendix to the following setting.

Example A.3. Let $Y$ be an $n$-dimensional finitely presented projective torsion scheme over $K^{\circ}$ for a nonArchimedean field $K$. For $q \in\{0, \ldots, n\}$, we define $h^{q}(Y, L) \in \mathbb{R}_{\geq 0}$ as the content of the torsion module $H^{q}(Y, L), c f$. Definition 3.8. It is shown in Lemma 3.19 that this function satisfies (A.1) and (A.2) with $s=n$, and therefore induces asymptotic cohomological functions $\hat{h}^{q}: \operatorname{Pic}(Y)_{\mathbb{R}} \rightarrow \mathbb{R}_{\geq 0}$. As in Example A.2, we will normalize $\hat{h}^{q}$ in this case with a factor $n$ !.

Lemma A.4. For any $L_{1}, \ldots, L_{r} \in P$, there exists $C>0$ such that

$$
\left|h\left(m_{1} L_{1}+\cdots+m_{r} L_{r}\right)-h\left(m_{1}^{\prime} L_{1}+\cdots+m_{r}^{\prime} L_{r}\right)\right| \leq C\left|m-m^{\prime}\right| \max \left\{|m|,\left|m^{\prime}\right|\right\}^{s-1}
$$

for all $m, m^{\prime} \in \mathbb{Z}^{r}$.

Proof. By (A.2) we can find $C>0$ such that

$$
\left|h\left(m_{1} L_{1}+\cdots+m_{r} L_{r} \pm L_{i}\right)-h\left(m_{1} L_{1}+\cdots+m_{r} L_{r}\right)\right| \leq C|m|^{s-1} .
$$

for all $m \in \mathbb{Z}^{r}$ and $i=1, \ldots, r$. An iterated application of this estimate yields the result.

Proof of Theorem A.1. Without loss of generality, we may assume that $P$ is finitely generated. Uniqueness is then clear, since (i) and (ii) uniquely determine $\hat{h}$ on $P_{\mathbb{Q}}$, which is dense in the finite dimensional vector space $P_{\mathbb{R}}$.

In a first step we show that $\tilde{h}: P \rightarrow \mathbb{R}$ defined by

$$
\tilde{h}(L):=\limsup _{m \rightarrow+\infty} m^{-s} h(m L)
$$


is $\mathbb{N}$-homogeneous of degree s, i.e.

$$
\tilde{h}(a L)=a^{s} \tilde{h}(L)
$$

for $a \in \mathbb{N}$ and $L \in P$. Note first that $\tilde{h}(L)$ is real-valued by (A.1). The case $a=0$ follows from $s>0$, and so we may assume $a \geq 1$. We obviously have

$$
\tilde{h}(L) \geq \limsup _{m \rightarrow+\infty}(a m)^{-s} h(a m L)=a^{-s} \tilde{h}(a L)
$$

Conversely, pick a sequence $m_{j} \rightarrow+\infty$ such that $m_{j}^{-s} h\left(m_{j} L\right) \rightarrow \tilde{h}(L)$, and write $m_{j}=a q_{j}+r_{j}$ with $q_{j} \in \mathbb{N}$ and $r_{j} \in\{0, \ldots, a-1\}$. Since $r_{j}$ takes only finitely many values, Lemma A.4 yields

$$
h\left(m_{j} L\right)=h\left(a q_{j} L\right)+O\left(m_{j}^{s-1}\right)
$$

and hence

$$
a^{s} \tilde{h}(L)=a^{s} \lim _{j} m_{j}^{-s} h\left(m_{j} L\right)=\lim _{j} q_{j}^{-s} h\left(a q_{j} L\right) \leq \tilde{h}(a L)
$$

which proves (A.4).

This first step yields that there is a unique function $\hat{h}: P_{\mathbb{Q}} \rightarrow \mathbb{R}$ which is $\mathbb{Q}_{+}$-homogeneous of degree $s$ and which satisfies (A.3). It remains to show that $\hat{h}$ extends continuously to $P_{\mathbb{R}}$. Pick $L_{1}, \ldots, L_{r} \in P$. By Lemma A.4, we have

$$
\left|h\left(m_{1} L_{1}+\cdots+m_{r} L_{r}\right)-h\left(m_{1}^{\prime} L_{1}+\cdots+m_{r}^{\prime} L_{r}\right)\right| \leq C\left|m-m^{\prime}\right| \max \left\{|m|,\left|m^{\prime}\right|\right\}^{s-1},
$$

for all $m, m^{\prime} \in \mathbb{Z}^{r}$. By homogeneity, this yields

$$
\left|\hat{h}\left(x_{1} L_{1}+\cdots+x_{r} L_{r}\right)-\hat{h}\left(x_{1}^{\prime} L_{1}+\cdots+x_{r}^{\prime} L_{r}\right)\right| \leq C\left|x-x^{\prime}\right| \max \left\{|x|,\left|x^{\prime}\right|\right\}^{s-1}
$$

for $x, x^{\prime} \in \mathbb{Q}^{r}$. As a result, $\hat{h}: P_{\mathbb{Q}} \rightarrow \mathbb{R}$ is uniformly continuous on each bounded subset of $P_{\mathbb{Q}}$, and hence admits a unique continuous extension to $P_{\mathbb{R}}$.

Proposition A.5. In the setting of Theorem A.1, pick $L_{1}, \ldots, L_{r} \in P$, and set for $x \in \mathbb{R}^{r}$ and $m \in \mathbb{N}$

$$
f_{m}(x):=m^{-s} h\left(\sum_{i}\left\lfloor m x_{i}\right\rfloor L_{i}\right) .
$$

(i) For all $x \in \mathbb{R}^{r}$, we have $\hat{h}\left(\sum_{i} x_{i} L_{i}\right)=\limsup _{m \rightarrow+\infty} f_{m}(x)$.

(ii) Assume given an open convex cone $\sigma \subset \mathbb{R}^{r}$ such that $\lim _{m \rightarrow+\infty} f_{m}(x)=\hat{h}(x)$ for all $x \in \sigma \cap \mathbb{Z}^{r}$. Then $\lim _{m \rightarrow+\infty} f_{m}(x)=\hat{h}(x)$ for all $x \in \bar{\sigma}$.

Proof. To prove (i), we set $\hat{h}(x):=\hat{h}\left(\sum_{i} x_{i} L_{i}\right)$ and $f(x):=\limsup _{m \rightarrow \rightarrow+\infty} f_{m}(x)$ for $x \in \mathbb{R}^{r}$. We note that $f(x)=\hat{h}(x)$ for $x \in \mathbb{Z}^{r}$, by (A.3).

By Lemma A.4, for all $m \in \mathbb{Z}_{>0}$ and $x, x^{\prime} \in \mathbb{R}^{r}$ with $|x|,\left|x^{\prime}\right| \leq R$ we have a uniform estimate

$$
\left|f_{m}(x)-f_{m}\left(x^{\prime}\right)\right| \leq C\left|x-x^{\prime}\right| \max \left\{|x|,\left|x^{\prime}\right|\right\}^{s-1}+O_{R}\left(m^{-1}\right) .
$$

This yields $\left|f(x)-f\left(x^{\prime}\right)\right| \leq C\left|x-x^{\prime}\right| \max \left\{|x|,\left|x^{\prime}\right|\right\}^{s-1}$, which shows that $f$ is continuous on $\mathbb{R}^{r}$. Arguing just as for (A.4), we further have $f(a x)=a^{s} f(x)$ for all $a \in \mathbb{Z}_{>0}$ and $x \in \mathbb{R}^{r}$. It follows that $f=\hat{h}$ on $\mathbb{Q}^{r}$, and hence also on $\mathbb{R}^{r}$, by continuity. This proves (i).

To prove (ii), we show in a first step that

$$
A:=\left\{x \in \mathbb{R}^{r} \mid \lim _{m \rightarrow \infty} f_{m}(x)=\hat{h}(x)\right\}
$$

is a closed subset of $\mathbb{R}^{r}$. Let us pick a sequence $\left(x_{n}\right)_{n \in \mathbb{N}}$ in $A$ converging to $x \in \mathbb{R}^{r}$. For $\varepsilon>0$, continuity of $\hat{h}$ yields that $\left|\hat{h}\left(x_{n}\right)-\hat{h}(x)\right|<\varepsilon / 3$ for $n \gg 0$. We choose such an $n$ which also satisfies

$$
C\left|x-x_{n}\right| \max \left\{|x|,\left|x_{n}\right|\right\}^{s-1}<\varepsilon / 6 .
$$


There is $m_{0} \in \mathbb{N}$ such that the term $O_{R}\left(m^{-1}\right)$ for $x^{\prime}=x_{n}$ in (A.5) is bounded by $\varepsilon / 6$ for all $m \geq m_{0}$ and all $n \in \mathbb{N}$. It follows from (A.5) that $\left|f_{m}\left(x_{n}\right)-f_{m}(x)\right|<\varepsilon / 3$ for $m \geq m_{0}$. Since $x_{n} \in A$, there is $m(n) \in \mathbb{N}_{\geq m_{0}}$ depending on $n$ such that $\left|f_{m}\left(x_{n}\right)-\hat{h}\left(x_{n}\right)\right|<\varepsilon / 3$ for all $m \geq m(n)$. Overall, the triangle inequality gives $\left|f_{m}(x)-\hat{h}(x)\right|<\varepsilon$ for all $m \geq m(n)$. This proves $\lim _{m \rightarrow \infty} f_{m}(x)=\hat{h}(x)$ and hence $x \in A$. We conclude that $A$ is closed.

By assumption, $A$ contains $\sigma \cap \mathbb{Z}^{r}$. By the first step, to prove (ii) it will thus be enough to show that $A$ contains $\sigma \cap \mathbb{Q}^{r}$, which is dense in the closed convex cone $\bar{\sigma}$. Let $x \in \sigma \cap \mathbb{Q}^{r}$, and pick $a \in \mathbb{Z}_{>0}$ such that $a x \in \mathbb{Z}^{r}$. For $m \in \mathbb{Z}_{>0}$, write $m=a q_{m}+r_{m}$ with $q_{m} \in \mathbb{N}$ and $r_{m} \in\{0, \ldots a-1\}$. Then $\left\lfloor m x_{i}\right\rfloor-q_{m} a x_{i}=\left\lfloor r_{m} x_{i}\right\rfloor$ remains bounded, and Lemma A.4 thus yields a constant $C^{\prime}>0$ depending on $|x|$ such that

$$
\left|h\left(\sum_{i}\left\lfloor m x_{i}\right\rfloor L_{i}\right)-h\left(q_{m} \sum_{i} a x_{i} L_{i}\right)\right| \leq C^{\prime} m^{s-1}
$$

for all $m \in \mathbb{Z}_{>0}$. Since $a x \in \sigma \cap \mathbb{Z}^{r}$, we have by assumption $q_{m}^{-s} h\left(q_{m} \sum_{i} a x_{i} L_{i}\right) \rightarrow \hat{h}(a x)=a^{s} \hat{h}(x)$. Using (A.6), we get $m^{-s} f_{m}(x) \rightarrow \hat{h}(x)$, i.e. $x \in A$, which concludes the proof of (ii).

\section{References}

[AB95] A. Abbes and T. Bouche, Théorème de Hilbert-Samuel "arithmétique”, Ann. Inst. Fourier (Grenoble) 45 (1995), no. 2, 375-401.

[Ber90] V. G. Berkovich, Spectral theory and analytic geometry over non-Archimedean fields, Mathematical Surveys and Monographs, vol. 33, American Mathematical Society, Providence, RI, 1990.

[BB10] R. Berman and S. Boucksom, Growth of balls of holomorphic sections and energy at equilibrium, Invent. Math. 181 (2010), no. 2, 337-394.

[BE21] S. Boucksom and D. Eriksson, Spaces of norms, determinant of cohomology and Fekete points in non-Archimedean geometry, Adv. Math. 378, article ID:107501 (2021).

[BFJ15] S. Boucksom, C. Favre, and M. Jonsson, Solution to a non-Archimedean Monge-Ampère equation, J. Amer. Math. Soc. 28 (2015), no. 3, 617-667.

[BGM20] S. Boucksom, W. Gubler, and F. Martin, Differentiability of relative volumes over an arbitrary non-Archimedean field, preprint arXiv:2004.03847 (2020). To appear in IMRN.

[BJ18] S. Boucksom and M. Jonsson, Singular semipositive metrics on line bundles on varieties over trivially valued fields, preprint arXiv:1801.08229 (2018).

$\left[\mathrm{BGJ}^{+} 20\right]$ J. I. Burgos Gil, W. Gubler, P. Jell, K. Künnemann, and F. Martin, Differentiability of nonArchimedean volumes and non-Archimedean Monge-Ampère equations (with an appendix by Robert Lazarsfeld), Algebr. Geom. 7 (2020), no. 2, 113-152.

[CL06] A. Chambert-Loir, Mesures et équidistribution sur les espaces de Berkovich, J. Reine Angew. Math. 595 (2006), 215-235.

[CD12] A. Chambert-Loir and A. Ducros, Formes différentielles réelles et courants sur les espaces de Berkovich, preprint arXiv:1204.6277 (2012).

[Che08] H. Chen, Positive degree and arithmetic bigness, preprint arXiv:0803.2583 (2008).

[Chell] _ Differentiability of the arithmetic volume function, J. Lond. Math. Soc. (2) 84 (2011), no. 2, 365-384.

[CM15] H. Chen and C. Maclean, Distribution of logarithmic spectra of the equilibrium energy, Manuscripta Math. 146 (2015), no. 3-4, 365-394. 
[CM18] H. Chen and A. Moriwaki, Extension property of semipositive invertible sheaves over a non-Archimedean field, Ann. Sc. Norm. Super. Pisa Cl. Sci. (5) 18 (2018), no. 1, 241-282.

[Gro60] A. Grothendieck, Éléments de géométrie algébrique. I. Le langage des schémas, Publ. Math. Inst. Hautes Études Sci. 4 (1960), 1-228.

[Gro61] _ Éléments de géométrie algébrique. II. Étude globale élémentaire de quelques classes de morphismes, Publ. Math. Inst. Hautes Études Sci. 8 (1961), 1-222.

[Gro66]__ Éléments de géométrie algébrique. IV. Étude locale des schémas et des morphismes de schémas. III, Publ. Math. Inst. Hautes Études Sci. 28 (1966), 1-255.

[Gub98] W. Gubler, Local heights of subvarieties over non-Archimedean fields, J. Reine Angew. Math. 498 (1998), 61-113.

[Gub03] _ Local and canonical heights of subvarieties, Ann. Sc. Norm. Super. Pisa Cl. Sci. (5) 2 (2003), no. $4,711-760$.

[Gub10] - Non-Archimedean canonical measures on abelian varieties, Compos. Math. 146 (2010), no. 3, 683-730.

[GK17] W. Gubler and K. Künnemann, A tropical approach to non-Archimedean Arakelov geometry, Algebra Number Theory 11 (2017), no. 1, 77-180.

[GM19] W. Gubler and F. Martin, On Zhang's semipositive metrics, Doc. Math. 24 (2019), 331-372.

[GRW17] W. Gubler, J. Rabinoff, and A. Werner, Tropical skeletons, Ann. Inst. Fourier (Grenoble) 67 (2017), no. 5, 1905-1961.

[GW10] U. Görtz and T. Wedhorn, Algebraic geometry I. Schemes. With examples and exercises, Advanced Lectures in Mathematics, Vieweg + Teubner, Wiesbaden, 2010.

[Har77] R. Hartshorne, Algebraic geometry, Springer-Verlag, New York-Heidelberg, Graduate Texts in Mathematics, no. 52, 1977.

[KT02] M. Kontsevich and Y. Tschinkel, Non-Archimedean Kähler geometry, unpublished note, 2002.

[Kür06] A. Küronya, Asymptotic cohomological functions on projective varieties., Am. J. Math. 128 (2006), no. $6,1475-1519$.

[Laz04a] R. Lazarsfeld, Positivity in algebraic geometry. I. Classical setting: line bundles and linear series, Ergebnisse der Mathematik und ihrer Grenzgebiete. 3. Folge, vol. 48, Springer-Verlag, Berlin, 2004.

[Laz04b] _ Positivity in algebraic geometry. II. Positivity for vector bundles, and multiplier ideals, Ergebnisse der Mathematik und ihrer Grenzgebiete. 3. Folge, vol. 49, Springer-Verlag, Berlin, 2004.

[Mor09] A. Moriwaki, Continuity of volumes on arithmetic varieties, J. Algebraic Geom. 18 (2009), no. 3, 407-457.

[Sch13] P. Scholze, p-adic Hodge theory for rigid-analytic varieties, Forum Math. Pi 1 (2013), el, 77pp.

[Stacks] The Stacks Project Authors, The Stacks Project, http://stacks.math.columbia.edu.

[Tem16] M. Temkin, Metrization of differential pluriforms on Berkovich analytic spaces, in: Non-Archimedean and tropical geometry (based on two Simons symposia, Island of St. John, March 31-April 6, 2013 and Puerto Rico, February 1-7, 2015), Simons Symposia, Springer, 195-285 (2016).

[Ul195] P. Ullrich, The direct image theorem in formal and rigid geometry, Math. Ann. 301 (1995), no. 1, 69-104.

[Yua08] X. Yuan, Big line bundles over arithmetic varieties, Invent. Math. 173 (2008), no. 3, 603-649. 
[YZ17] X. Yuan and S.-W. Zhang, The arithmetic Hodge index theorem for adelic line bundles, Math. Ann. 367 (2017), no. 3-4, 1123-1171.

[Zha95] S.-W. Zhang, Small points and adelic metrics, J. Algebraic Geom. 4 (1995), no. 2, 281-300. 